\title{
Weak Fault Signal Detection of Rotating Machinery Based on Multistable Stochastic Resonance and VMD-AMD
}

\author{
Dongying Han $\mathbb{D},{ }^{1}$ Xiao Su, ${ }^{2}$ and Peiming Shi $\mathbb{D}^{2}$ \\ ${ }^{1}$ School of Vehicles and Energy, Yanshan University, Qinhuangdao, Hebei 066004, China \\ ${ }^{2}$ School of Electrical Engineering, Yanshan University, Qinhuangdao, Hebei 066004, China \\ Correspondence should be addressed to Peiming Shi; peiming.shi@163.com
}

Received 29 November 2017; Revised 24 January 2018; Accepted 7 March 2018; Published 12 April 2018

Academic Editor: L. Zanotti Fragonara

Copyright (C) 2018 Dongying Han et al. This is an open access article distributed under the Creative Commons Attribution License, which permits unrestricted use, distribution, and reproduction in any medium, provided the original work is properly cited.

For solving detection problems of multifrequency weak signals in noisy background, a novel weak signal detection method based on variational mode decomposition (VMD) and rescaling frequency-shifted multistable stochastic resonance (RFMSR) with analytical mode decomposition (AMD) is proposed. In this method, different signal frequency bands are processed by rescaling subsampling compression to make each frequency band meet the conditions of stochastic resonance. Before the enhanced signal components are synthesized, they are processed to achieve the enhanced signal by means of AMD, leaving only the enhanced sections of the signal. The processed signal is decomposed into intrinsic mode functions (IMF) by VMD, in order to require the detection of weak multifrequency signals. The experimental analysis of the rolling bearing inner ring fault and gear fault diagnosis demonstrate that the proposed method can not only enhance signal amplitude, reduce false components, and improve the VMD algorithm's accuracy, but also effectively detect weak multifrequency signals submerged by noise.

\section{Introduction}

Because rotating machinery has come to play an increasingly significant role in transportation and industrial production, mechanical faults and damage, particularly bearing and gear faults, have recently been the cause of greater catastrophes and losses. Therefore, an accurate health monitoring and diagnosis system is required to identify incipient faults that may occur in rotating machines. However, two challenging issues exist in vibration signal analysis: nonstationary collected signals and signals that are usually mingled with heavy noise as a result of coupled machine components and the working environment $[1,2]$. Advanced signal processing techniques have been extensively developed for machine fault feature extraction, voice recognition, image processing techniques, leak detection in pipelines [3-5], and so on. Established techniques include wavelet transform [6], timefrequency distribution (TFD) [7], Hilbert-Huang transform (HHT) [8], blind source separation [9], and multivariate statistical analysis [10]. In summary, signal detection technology can be divided into two concepts [11-13]. The first involves obtaining a useful signal by eliminating or suppressing noise. Standard techniques, such as the wavelet denoising method, analytical mode decomposition (AMD), empirical mode decomposition (EMD) method, ensemble empirical mode decomposition (EEMD), local mean decomposition (LMD) method, and variational mode decomposition (VMD), inevitably weaken the useful signal while removing noise. For example, in the wavelet denoising method, the appropriate wavelet basis needs to be selected; if selection of the wavelet basis is improper, the final result will differ from the original signal. EMD exhibits a boundary effect and produces many false mode components, which has an impact on signal detection results [14-16]. EEMD, developed to overcome the mode mixing of EMD in [17], must be conducted numerous times, which inevitably results in a large computational burden [18]. Meanwhile, LMD methods are still recursive and data-driven and never consider the form of fault features. As a new adaptive decomposition method, VMD, which is also based on the concept of IMF, was proposed by Dragomiretskiy and Zosso [19]. It has been pointed out that VMD is theoretically much better founded than the sequential iterative sifting of EMD, because VMD is based on a clear variational model, and the resulting 


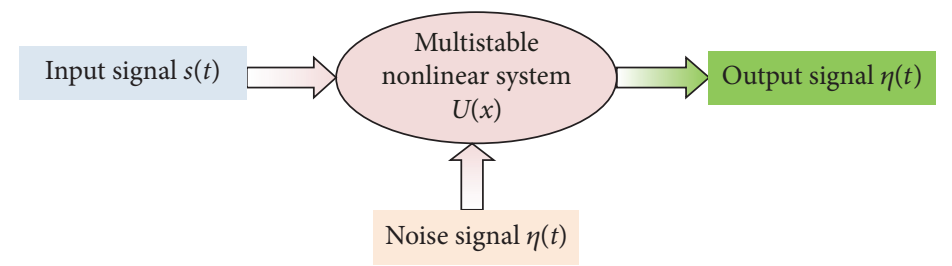

FIGURE 1: Multistable nonlinear system.

minimization steps perform concurrent mode extraction intuitively. As a result of noniterative decomposition and adaptively selected bands, VMD has the ability to overcome the mode mixing and misclassification problems caused by fixed band allocation [20]. Furthermore, much of the research $[21,22]$ has indicated that VMD, which can decompose the nonstationary signal into a couple of intrinsic mode functions adaptively and nonrecursively, offers a feasible tool. However, VMD is still not appropriate for analysis of a vibration signal with strong background noise [23], and the presence of strong noise in the measured vibration signal is unavoidable.

The second detection method is stochastic resonance (SR), which can detect signals by using the noise instead of removing it. Since the SR concept was proposed by Benzi et al. in 1981 [24], it has been widely applied in signal processing, physics, biology, large mechanical fault diagnosis, and other fields [25-33]. A weak periodic signal SR method has been proposed in locally optimal processors with a Fisher information metric [34]. Tan et al. realized SR with large parameters by means of subsampling [35]. Leng et al. [36] developed methods for transforming a high frequency into a low frequency, based on frequency rescaling or modulation, in order to satisfy the traditional SR requirements. These studies provide methods for the application of SR technology in processing large parameters signals. However, the majority of research has been based on the bistable SR system. The noise metastatic capacity of a multistable SR system is superior to that of the bistable SR system; therefore, using the multistable SR method to obtain frequency provides greater accuracy [37]. Meanwhile, the rotating machine's defectinduced fault signal is often corrupted by noise from other coupled machine components and the working environment, which makes certain incipient faults difficult to recognize. In particular, for the detection problem of weak multifrequency noisy signals under the influence of strong noise, extracting useful information is highly challenging, and has important practical significance.

In this paper, a novel method is proposed based on VMD after denoising, by means of rescaling frequencyshifted multistable SR with AMD. The remainder of this paper is organized as follows. Section 2 provides a brief introduction to the principles of multistable SR and the AMD algorithm, as well as introducing the principle of VMD and the limitations of the method in practical applications. Section 3 presents the application examples for the bearing and gears. Finally, conclusions are outlined in Section 4.

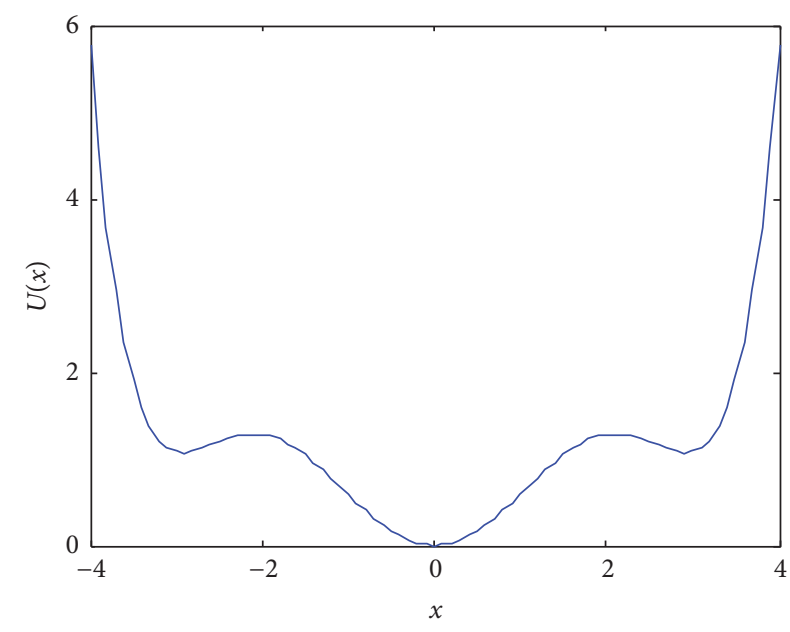

FIGURE 2: The multistable potential function $U(x)$.

\section{Multistable SR with Analytical Mode Decomposition}

2.1. Principles of Multistable SR. The model for multistable SR consists of a multistable nonlinear system, driven by periodic signals and Gaussian noise, which is shown in Figure 1. The Langevin equation can be obtained as follows [38]:

$$
\frac{\mathrm{d} x}{\mathrm{~d} t}=-\frac{\mathrm{d} U(x)}{\mathrm{d} x}+s(t)+\eta(t)
$$

where $s(t)=\sum_{i=1}^{n} A_{i} \sin \left(2 \pi f_{i} t\right)$ is the input signal with periodic signal amplitude $A_{i}$ and driving frequency $f_{i}, \eta(t)=$ $\sqrt{2 D} \varepsilon(t)$. Furthermore, $\langle\eta(t) \eta(t+\tau)\rangle=2 D \varepsilon(t)$ denotes the noise item, in which the $D$ is the noise intensity and $\varepsilon(t)$ represents the Gaussian white noise with a zero mean and unit variance.

Based on the structural characteristics of current resonance models, the reflection-symmetric sextic potential function $U(x)$ in (1) is defined as [37]

$$
U(x)=\frac{x^{6}}{6 a^{\prime}}-\frac{(1+c) x^{4}}{4 b^{\prime}}+\frac{c}{2} x^{2},
$$

where $a^{\prime}, b^{\prime}$, and $c$ denote the barrier parameters of the multistable potential with positive real values and $b^{\prime}=5$, $c>0, a^{\prime}=20+5 c(0<c<1), a=27.5-2.5 c(1<c<3)$. The above equation has three stable and two unstable solutions, as shown in Figure 2. 


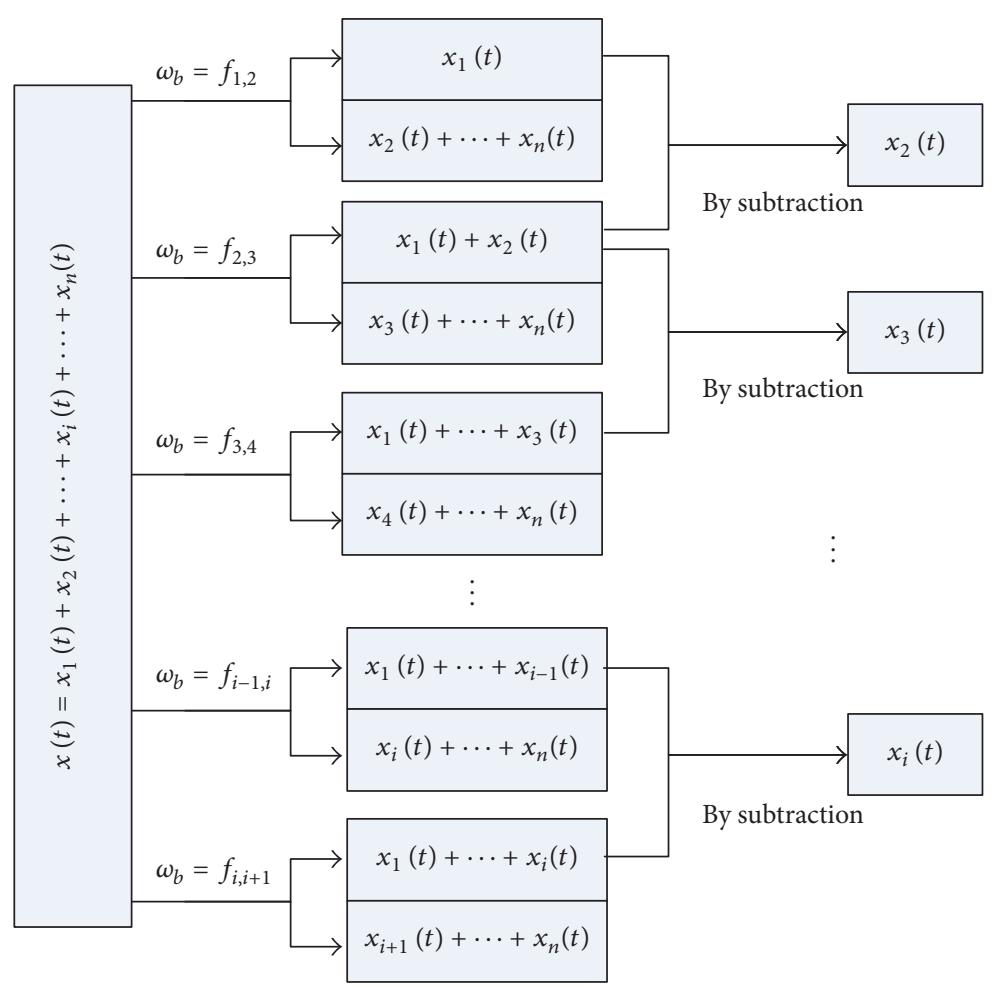

FIGURE 3: The schematic diagram of the extracted signal by AMD method.

Meanwhile, (2) can be rewritten as

$$
\begin{aligned}
\frac{\mathrm{d} x}{\mathrm{~d} t}= & -\frac{x^{5}}{a^{\prime}}+\frac{(1+c)}{b^{\prime}} x^{3}-c x+\sum_{i=1}^{n} A_{i} \sin \left(2 \pi f_{i} t\right) \\
& +\eta(t) .
\end{aligned}
$$

When $\sum_{i=1}^{n} A_{i} \sin \left(2 \pi f_{i} t\right)+\eta(t)=0$, the potential energy is at a minimum and the system is most stable. When a weak periodic signal and noise are input into the system, the noise energy is partially transferred to the signal to produce interactions; the output signal amplitude is greater than that of the input signal, which illustrates the SR phenomenon.

2.2. Brief Introduction to AMD Algorithm. A signal decomposition method known as AMD was proposed by Chen and Wang in 2012 [39]. This method can decompose signals with closely spaced frequency components, which results in the ability to extract the signal [40]. Furthermore, Feldman [41] presented a proof with a new interpretation of the formula, using the Bedrosian identity for overlapping signals, and further explained the decomposition method, which can be used as a low-pass filter. Because the AMD method can decompose a time series into the form of any two signals, it can not only realize the low-pass filtering function, but also extract the signal on any frequency components. However, the key to solving this problem is knowledge of the original signal's frequency components. Suppose a time series exists $x(t)=x_{1}(t)+x_{2}(t)+\cdots+x_{n}(t)$, with frequencies $f_{1}, f_{2}, \ldots, f_{n}$, respectively, and $f_{1}<f_{2}<\cdots<f_{n}$. If we extract $f_{n}$ frequency components of the signal, we can simply take the value between $f_{n}$ and $f_{n+1}$ as the two-partitioning frequency to decompose. Figure 3 illustrates a schematic diagram of the extracted signal, and the details of the AMD algorithm can be obtained from [39].

2.3. Brief Introduction to VMD. As a new, adaptive, and quasi-orthogonal signal decomposition method, VMD aims to decompose a real-valued signal $f$ into a discrete number of subsignals $u_{k}$ with certain sparsity properties, and all subsignals are mostly compact around a centre pulsation $\omega_{k}$. Therefore, VMD can be regarded as a constrained variational problem, as given in (4) [19]:

$$
\begin{array}{ll}
\min _{u_{k}, \omega_{k}} & \left\{\sum_{k}\left\|\partial_{t}\left[\left(\delta(t)+\frac{j}{\pi t}\right) * u_{k}(t)\right] e^{-j \omega_{k} t}\right\|_{2}^{2}\right\} \\
\text { s.t. } \quad & \sum_{k} u_{k}=f .
\end{array}
$$

In (4), $u_{k}$ represents the sub-signals and $\omega_{k}$ the centre frequencies of the submodes, $\alpha$ denotes the penalty parameter, and $\delta(t)$ is the Dirac distribution. In order to render this problem unconstrained, the quadratic penalty term and Lagrangian multipliers are incorporated, and the problem is rewritten as

$$
\begin{aligned}
L\left(u_{k}, \omega_{k}, \lambda\right)= & \alpha \sum_{k}\left\|\partial_{t}\left[\left(\delta(t)+\frac{j}{\pi t}\right) * u_{k}(t)\right] e^{-j \omega_{k} t}\right\|_{2}^{2} \\
& +\left\|f-\sum u_{k}\right\|_{2}^{2}+\left\langle\lambda, f-\sum u_{k}\right\rangle .
\end{aligned}
$$




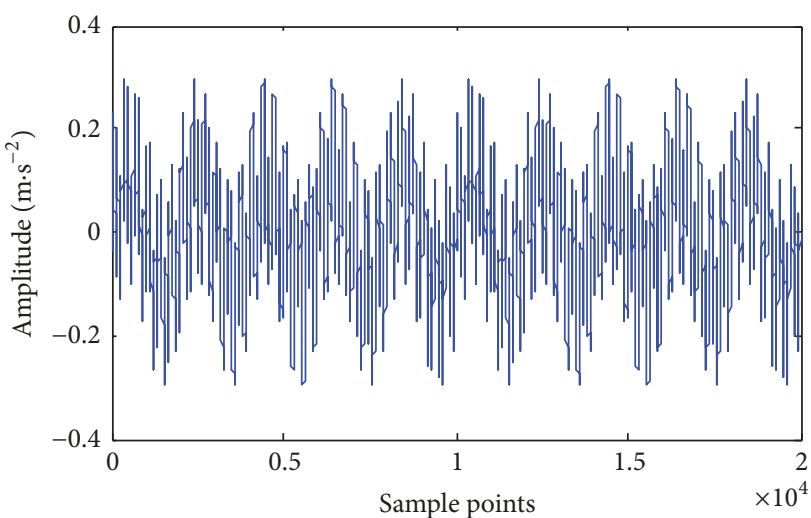

(a)

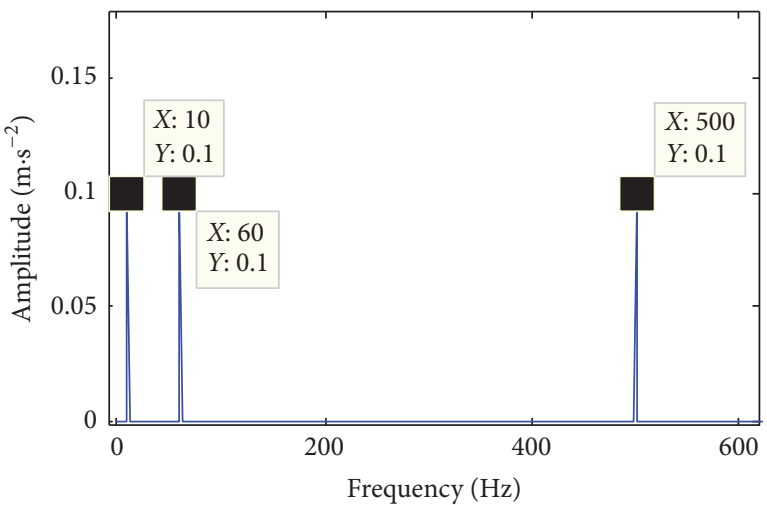

(b)

Figure 4: The time domain and spectrum of the simulated signal with the $D=0$.

Eq. (5) can be solved by means of the alternate direction method of multipliers (ADMM) [42]. Firstly, the mode number $k$ is determined artificially, while the frequency domain expression of mode function $\widehat{u}_{k}^{1}$, the centre frequency of each mode $\omega^{1}{ }_{k}$, and the Lagrangian multiplier $\hat{\lambda}^{1}$ are initialized. Then, modes $u_{k}$ and their centre frequency $\omega_{k}$ are updated by (6) and (7), respectively:

$$
\begin{aligned}
& \widehat{u}_{k}^{n+1} \longleftarrow \frac{\widehat{f}-\sum_{i<k} \widehat{u}_{i}^{n+1}-\sum_{i>k} \widehat{u}_{i}^{n+1}+\widehat{\lambda}^{n} / 2}{1+2 \alpha\left(\omega-\omega_{n}{ }^{k}\right)^{2}}, \\
& \omega_{k}^{n+1} \longleftarrow \frac{\int_{0}^{\infty} \omega\left|\hat{u}_{k}^{n+1}(\omega)\right|^{2} \mathrm{~d} \omega}{\int_{0}^{\infty}\left|\widehat{u}_{k}^{n+1}(\omega)\right|^{2} \mathrm{~d} \omega} .
\end{aligned}
$$

Following the updating of modes and centre frequencies, the Lagrangian multiplier $\widehat{\lambda}$ is also renewed by

$$
\hat{\lambda}^{n+1} \longleftarrow \widehat{\lambda}^{n}+\tau\left(\widehat{f}-\sum_{k} \widehat{u}_{k}^{n+1}\right)
$$

The updating is executed iteratively until the convergent Eq. (9) is satisfied, and

$$
\frac{\sum_{k}\left\|\widehat{u}_{k}^{n+1}-\widehat{u}_{k}^{n}\right\|_{2}^{2}}{\left\|\hat{u}_{k}^{n}\right\|_{2}^{2}}<\varepsilon .
$$

IMFs based on the preset mode number are obtained.

2.4. Limitations of VMD in Feature Extraction. As VMD regards the mode as an AM-FM signal and allocates modes adaptively in the frequency domain, it can effectively extract the characteristic frequency. In order to verify the efficiency of the VMD method, three sine signals are selected as the simulated signals. The test signals are generated according to the following equation:

$$
\begin{aligned}
x(t)= & 0.1 \times \sin (2 \pi \times 10 \times t)+0.1 \\
& \times \sin (2 \pi \times 60 \times t)+0.1 \times \sin (2 \pi \times 500 \times t) \\
& +\eta(t),
\end{aligned}
$$

where $\eta(t)=\sqrt{2 D} \delta(t)$ represents the noise item, in which $D$ is the noise intensity and $\delta(t)$ denotes Gaussian white noise with zero mean and unit variance. In order for this signal to consist of three different frequencies, the value of $k$ is set to 3 . At first, we set the value of $D=0$. Figure 4 illustrates the simulated signal waveforms, where the labels (a) and (b) represent the time domain and spectrum waveform, respectively. Subsequently, we decompose the simulated signal by using VMD and show the decomposition result in Figure 5, where we note that the VMD method accurately distinguishes the mixed sine signals in the spectrum. Furthermore, we set the value of $D$ to 2, in order to observe the robustness of the VMD method for mixed sine signals. The result of the simulated signals is presented in Figure 6, which indicates that the characteristic frequency is submerged in the heavy noise background. It can be seen from Figure 7 that the characteristic frequency is submerged by noise in the spectrum of IMFs, which means that the VMD method cannot fully identify the feature frequency hidden in the heavy noise background, although the VMD method decomposition is based on an accurate mode number. Therefore, we can conclude that even if the mode number is accurately preset, the feature frequency is still difficult to extract from the heavy background noise. Therefore, signal processing is required for noise reduction prior to VMD decomposition.

\section{Method for Weak Multifrequency Signal Detection Based on VMD following Denoising by RFMSR with AMD}

3.1. Detailed Implementation Steps of Proposed Method. It can be observed that although the VMD method can effectively extract fault features from the multifrequency signal, heavy background noise may lead to information loss. Meanwhile, improved mode number selection of mode number plays an important role in avoiding weak fault features submerged by noise [19]. In order to overcome these constraints, we propose a method based on the VMD following denoising by RFMSR with $\mathrm{AMD}$, for better extracting the characteristic frequency and to observe clearly the VMD decomposition results. In 

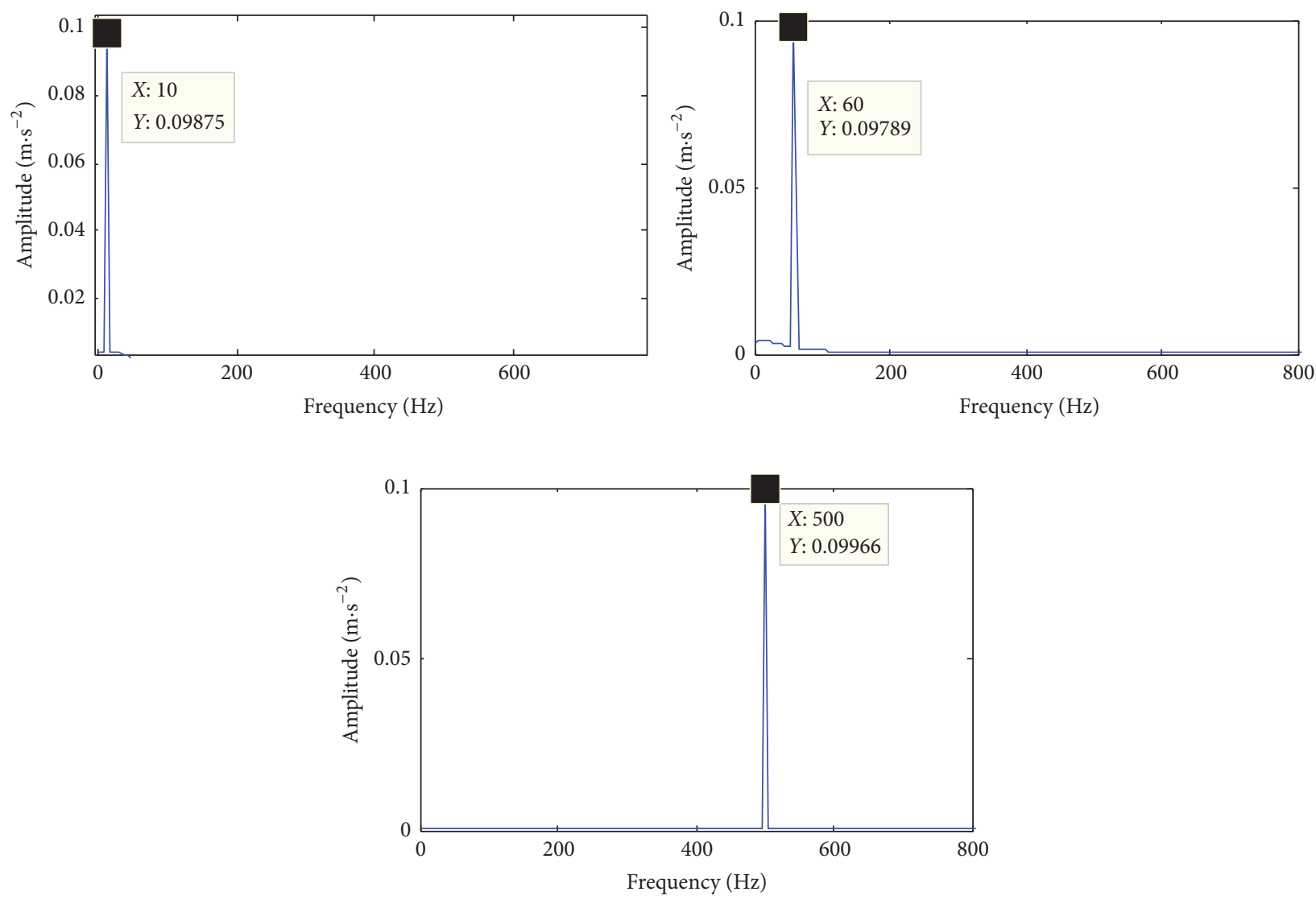

FIgURE 5: The spectrum of three IMFs by VMD with $D=0$.

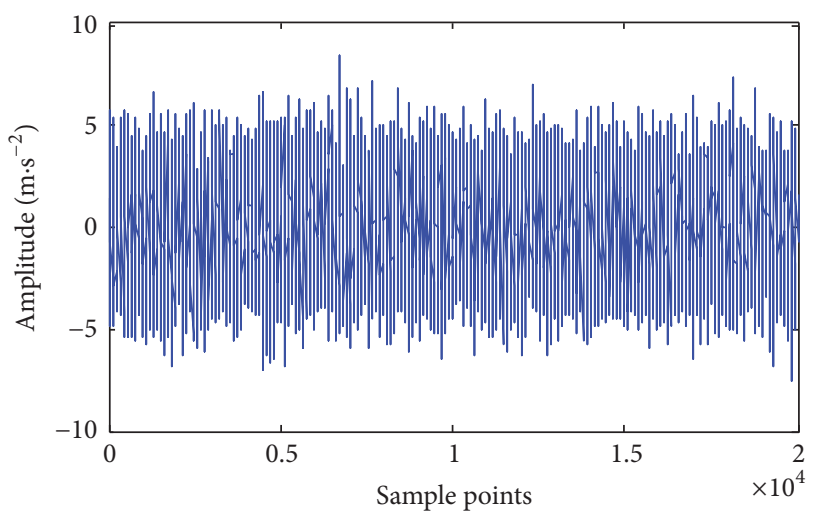

(a)

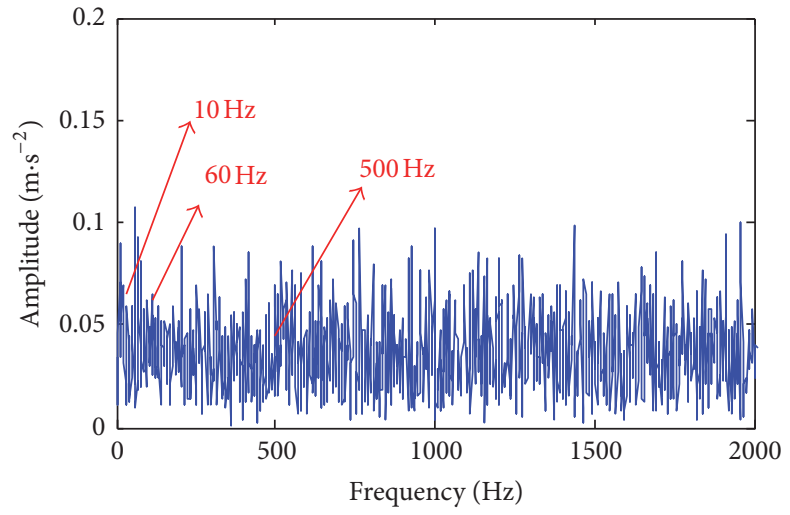

(b)

FIGURE 6: The time domain and spectrum of noisy signal.

order to obtain the better selection of mode number $k$ and penalty parameter $\alpha$, this paper adopted the method proposed by [23]. We use the genetic algorithm $[43,44]$ to search the best value and regard the envelope spectrum entropy of the intrinsic mode as the fitness value of the optimization process. The envelope spectrum entropy minimization is the final goal of optimization. The faults in bearings and gears usually are indicated as periodic impact features, which can be detected through the envelope spectrum of a given signal. Therefore, the envelope spectrum entropy value of intrinsic mode components obtained by VMD can be selected as the fitness value. For a given signal $x(t)$, its envelope spectrum entropy value $E_{p}$ can be described as follows:

$$
\begin{gathered}
E_{p}=-\sum_{j=1}^{N} p_{j} \ln \left(p_{j}\right), \\
p_{j}=a_{j}(t) l \sum_{j=1}^{N} a(j),
\end{gathered}
$$



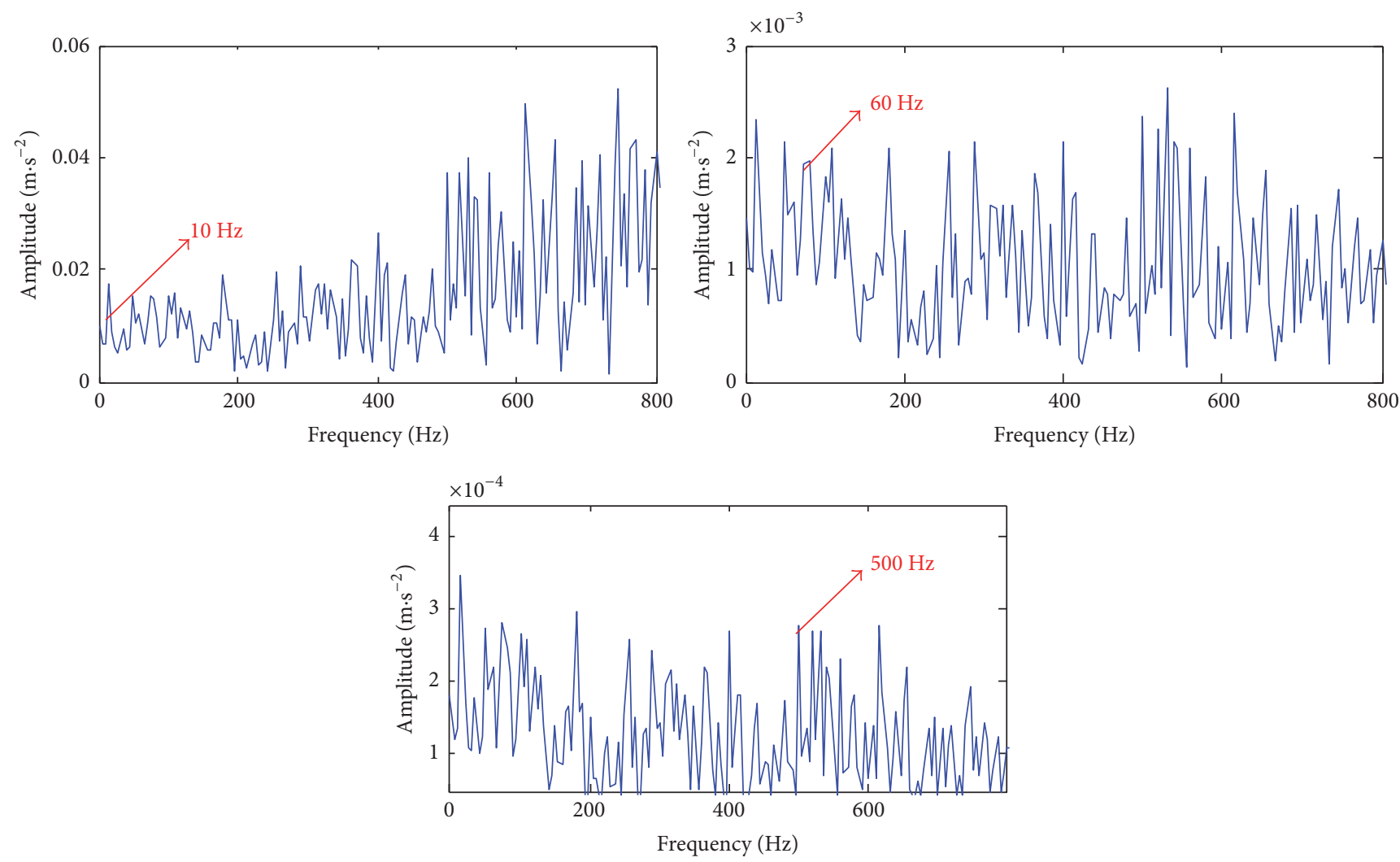

FIGURE 7: The spectrum of three IMFs through VMD with noise intensity $D=2$.

$$
\begin{aligned}
\hat{x}(t) & =\frac{1}{\pi} \int_{-\infty}^{+\infty} \frac{x(t)}{t-\tau} \mathrm{d} \tau, \\
z(t) & =x(t)+j \widehat{x}(t)=a_{j}(t) e^{j \theta(t)}, \\
a_{j}(t) & =\sqrt{x^{2}(t)+\hat{x}^{2}(t),} \\
\theta(t) & =\arctan \frac{x(t)}{\hat{x}(t)},
\end{aligned}
$$

where $a(j)$ represents the envelope of the given signal $x(t)$, which can be obtained from (13), $p_{j}$ is the normalized form of envelope $a(j), \hat{x}(t)$ is the Hilbert transform results of the given signal $x(t), z(t)$ is the analytic signal, and $\theta(t)$ is the instantaneous phase. According to the information theory, the strongest sparse characteristics have the minimum entropy value. That is, the smaller the size of the envelope spectrum entropy $E_{p}$ is, the clearer the distribution of signal sequence will be. Therefore, in this study, the minimum envelope spectrum entropy value (MESEV) of intrinsic mode components is regarded as the fitness value of the genetic algorithm, and it can be expressed as follows:

$$
\min \left\{E_{P}\right\}=\left\{E_{P 1}, E_{P 2}, \ldots, E_{P k}\right\},
$$

where $k$ is the number of intrinsic mode components obtained by VMD and $E_{P k}$ represents the envelope spectrum entropy value of the $k$ th intrinsic mode components. Consequently, we obtain the optimal decomposition parameters, regarding the minimization of MESEV as the ultimate optimization goals. That is, when MESEV becomes the smallest, the optimal decomposition parameters $\alpha$ and mode number $k$ can be obtained. In this paper, we generate the initial population $p=\left[p_{1}, p_{2}, \ldots, p_{L}\right]$. In this process, the decomposition parameters $\alpha$ and $k$ are subject to binary coding, and the encoded $\alpha$ and $k$ form the individuals $P_{i}$ $(i=1,2, \ldots, L)$. The population scale $L$ and the number of iterations are set as 50 and 30, respectively. And the crossover probability and aberration rate are set as 0.05 and 0.7 . In order to improve the computational efficiency and precision, the value range for parameters $\alpha$ and $k$ is set to $[100,2000]$ and $[2,10]$, respectively. Carry out the procedure of selection, crossover, and mutation and use new individuals to update the population until obtaining the minimization of MESEV, so that the mode number $k$ could be obtained. Moreover, fault feature frequency in the theory of bearings and gears can be calculated prior to the experiment, which greatly promotes application of the AMD method. The steps of the proposed method are as follows:

(1) Use the genetic algorithm to search the best value and regard the envelope spectrum entropy of the intrinsic mode as the fitness value of the optimization process. The envelope spectrum entropy minimization is the final goal of optimization. So the number of characteristic frequencies can be obtained.

(2) A frequency band transform of the sampling frequency of the noisy signal is carried out, in order to satisfy the SR small parameter condition in the two sampling frequencies. 


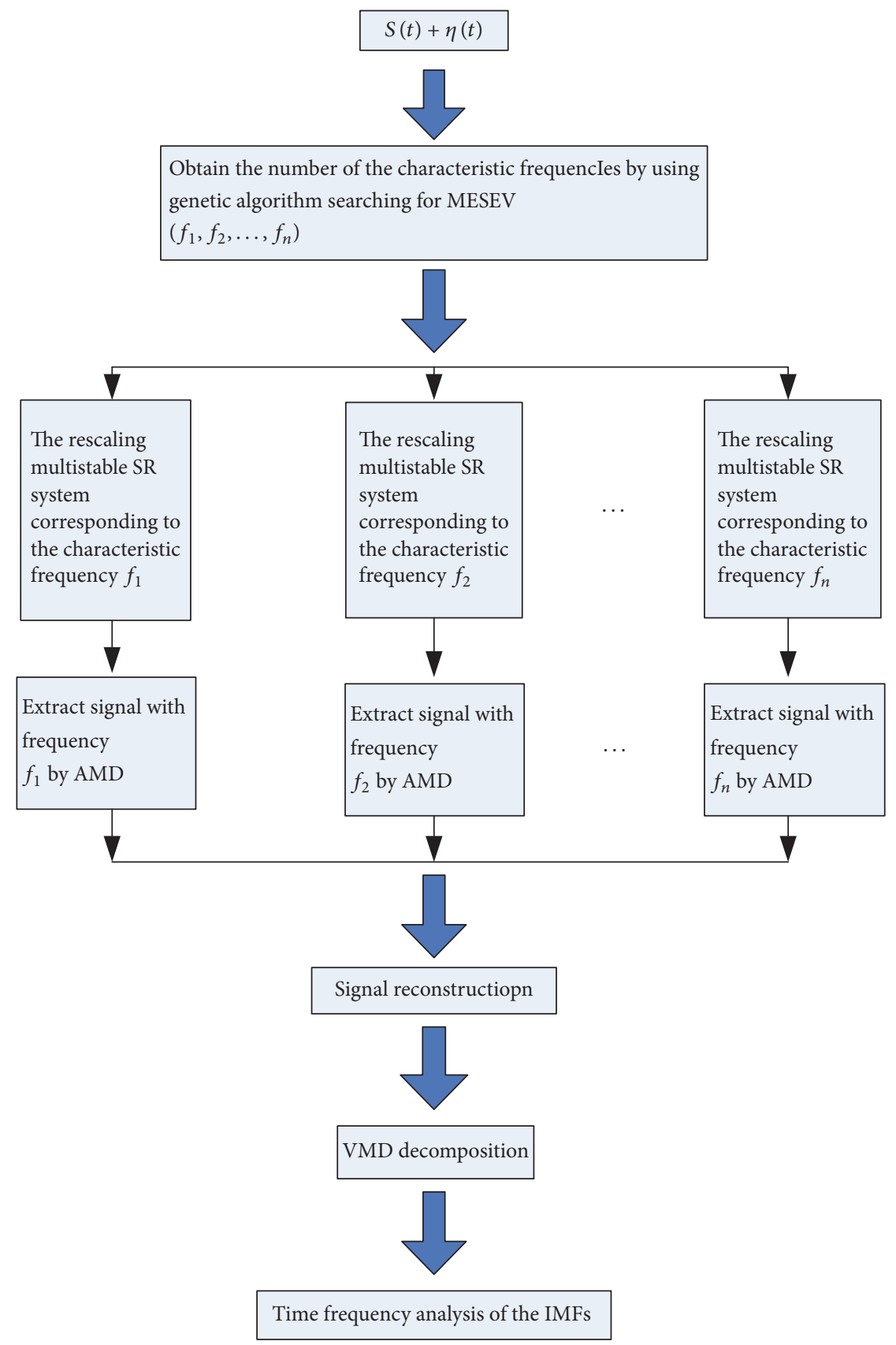

FIGURE 8: The flow process diagram of proposed method.

(3) The transformed signal passes through the multistable SR system to enhance the frequency band's useful signal.

(4) The random resonance output signal is restored.

(5) The AMD method is used to restore the signal, and only the signal components of the band are reserved.

(6) The steps are repeated for different frequency bands (n) of the original signal (1)-(5).

(7) The enhancement signals of the different frequency bands are synthesized to obtain an enhanced signal to realize the weak multifrequency signal measurement.

The flow process diagram for the proposed method can be seen in Figure 8. As opposed to [34], which is based on improving the VMD itself, the proposed method is based on the multistable SR and AMD method can be considered as an improved pretreatment method prior to VMD decomposition. Furthermore, the combination of the RFMSR with AMD and VMD takes full advantage of each method, increasing its practicality and effectiveness.

3.2. The Simulation Validation. In Section 2.4, it was noted that the primal VMD method cannot effectively extract the feature frequency from a noisy signal; therefore, we apply the improved method to address this problem. On this occasion, we still use the simulated noisy signal structured 


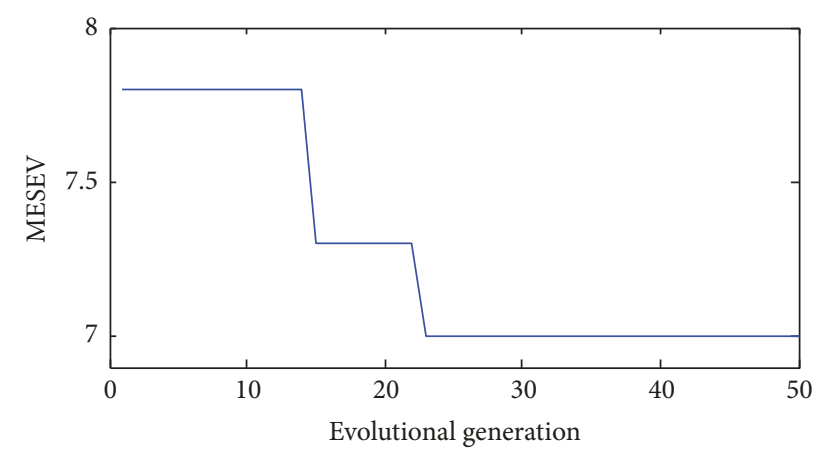

FIgURE 9: The relation curve between MESEV and Evolutional generation.

in Section 2.4, and the mixed signal waveform is shown in Figure 6(a), in which we can hardly determine the characteristic frequency. The spectrum in Figure 6(b) also reveals little information about the feature frequency; therefore, the improved method is applied. The relation curve of the MESEV changing with evolutional generation is depicted in Figure 9. It can be seen from Figure 9 that the MESEV is the steadiest and smallest when the evolutional generation is gradually increased from 23 to 50 . Consequently, according to the evolution results of the genetic algorithm as shown in Figure 9, the optimal decomposition parameters $\alpha$ and $k$ of VMD are set as 1800 and 3, respectively. According to the decomposition $k$, we can assume three characteristic frequencies with $f_{1}, f_{2}$, and $f_{3}$, respectively, and we then process the RFMSR on each frequency range in order to improve the signal intensity of $f_{1}, f_{2}$, and $f_{3}$, respectively. It should be noted that the parameter $c$ of the multistable model is set to 0.2 and the double sample frequencies are set to 3,1 , and $0.8 \mathrm{~Hz}$, respectively. The enhancement effect of the rescaling frequency-shifted SR can be observed in Figure 10. It can be seen from Figure 10(a) that certain highfrequency components are weakened, while the amplitude of the corresponding frequency bands is greatly improved. The amplitude of the characteristic frequency of $10 \mathrm{~Hz}$ increases from 0.1 to 0.7133 , that of $60 \mathrm{~Hz}$ increases from 0.1 to 0.5183 in Figure 10(b), and that of $500 \mathrm{~Hz}$ increases from 0.1 to 0.346 in Figure 10(c). Although the amplitudes of the three feature frequencies in Figures 10(a), 10(b), and 10(c) are enhanced, they are still weak; therefore, they are difficult to detect in the output signal. Moreover, certain interference frequencies exist around the fault frequency, which means that at times the weak multifrequency signals cannot be detected using multistable SR alone. Next, the three output signals through the multistable system are, respectively, dealt with by means of the AMD method. The different frequency band signals are selected by the AMD method, and each resonance band is retained only after AMD processing. The time domain plot of synthetic signals in Figure 11(a) has been obtained. Finally, the synthesized signal is decomposed by means of VMD, in order to obtain an ideal frequency enhancement effect, as shown in Figures 11(b)-11(d), which effectively demonstrates that simulated signal frequencies exist. Therefore, this study verified the effectiveness of the method.
TABLE 1: The main parameters of the rolling bearings.

\begin{tabular}{lc}
\hline Inner diameter & $17.0002(\mathrm{~mm})$ \\
Outer diameter & $39.9999(\mathrm{~mm})$ \\
Pitch diameter & $28.4988(\mathrm{~mm})$ \\
Ball diameter & $6.7462(\mathrm{~mm})$ \\
Ball number & 8 \\
Contact angle $/\left(^{\circ}\right)$ & 0 \\
\hline
\end{tabular}

TABLE 2: Rolling bearing fault feature frequency $\left(f_{r}=29.95 \mathrm{~Hz}\right)$.

\begin{tabular}{lc}
\hline Bearing element & Failure frequency \\
\hline Inner ring & $4.9469 f_{r}$ \\
Outer ring & $3.0530 f_{r}$ \\
The retainer & $0.3817 f_{r}$ \\
Rolling body & $3.9874 f_{r}$ \\
\hline
\end{tabular}

\subsection{Application of Proposed Method}

3.3.1. Analysis of Rolling Bearing. Rolling bearing fault signals are typically nonstationary, modulated, and weak, making it challenging to detect and extract feature information, which is often submerged in strong background noise. The data used in this study are taken from the Case Western Reserve University (CWRU) Bearing Data Centre. As illustrated in Figure 12, the apparatus for data acquisition consists of a $1.5 \mathrm{kw}$ motor, torque transducer, dynamometer, and control electronics (not shown). The IEPE Accelerometer is adopted with linear frequency band from 0.7 to $13,000 \mathrm{~Hz}$ in this paper.

Two deep-groove ball bearings support the motor shaft at the drive and fan ends of the motor. Single-point defects are set on the test bearings at the location of the outer raceway, inner raceway, and rolling element, using electrodischarge machining. The vibration data were taken from the fan end of the bearing (type 6203-2RS JEM SKF) with the inner race defects, by using an accelerometer attached to the motor housing at the drive end. The bearings used in this test are deep-groove ball bearings of type 6203-2RS JEM SKF, the motor speed is $n=1797 \mathrm{rpm}\left(f_{r}=29.95 \mathrm{~Hz}\right)$, and the sampling frequency is $12 \mathrm{kHz}$. The geometric details of this bearing type are provided in Table 1, while the characteristic frequencies are displayed in Table 2. Taking the inner raceway faults as an example, calculating the feature frequencies results in $148.2 \mathrm{~Hz}$; twice this is $296.4 \mathrm{~Hz}$ and triple is $444.6 \mathrm{~Hz}$.

Figure 13 illustrates the time domain and spectrum of the rolling bearing inner race fault and its partial amplified graph. In Figure 13, we label the respective fault characteristic frequencies in order to compare these with the output signal through the multistable system. It can be seen that the time domain waveform of the signal periodic impact vibration is very obvious. However, the spectrum energy is distributed in a wide frequency range, rather than in the low-frequency vibration characteristics, causing the fault characteristic frequencies to be extremely weak. According to the proposed method, firstly, genetic algorithm is adopted to acquire the 

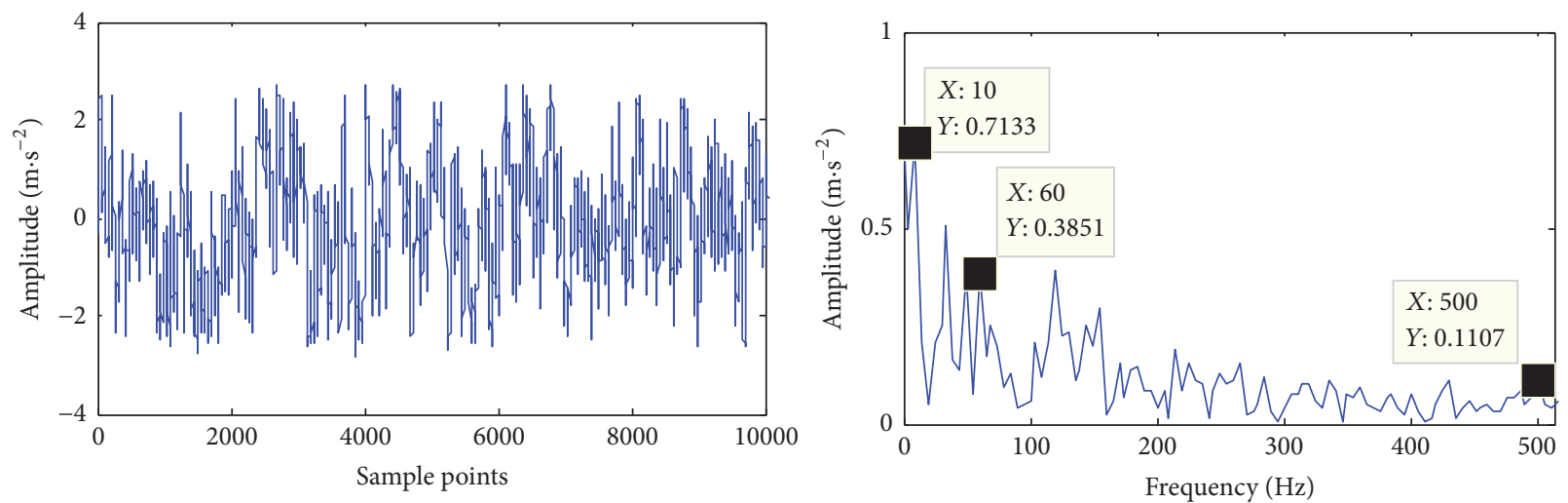

(a)
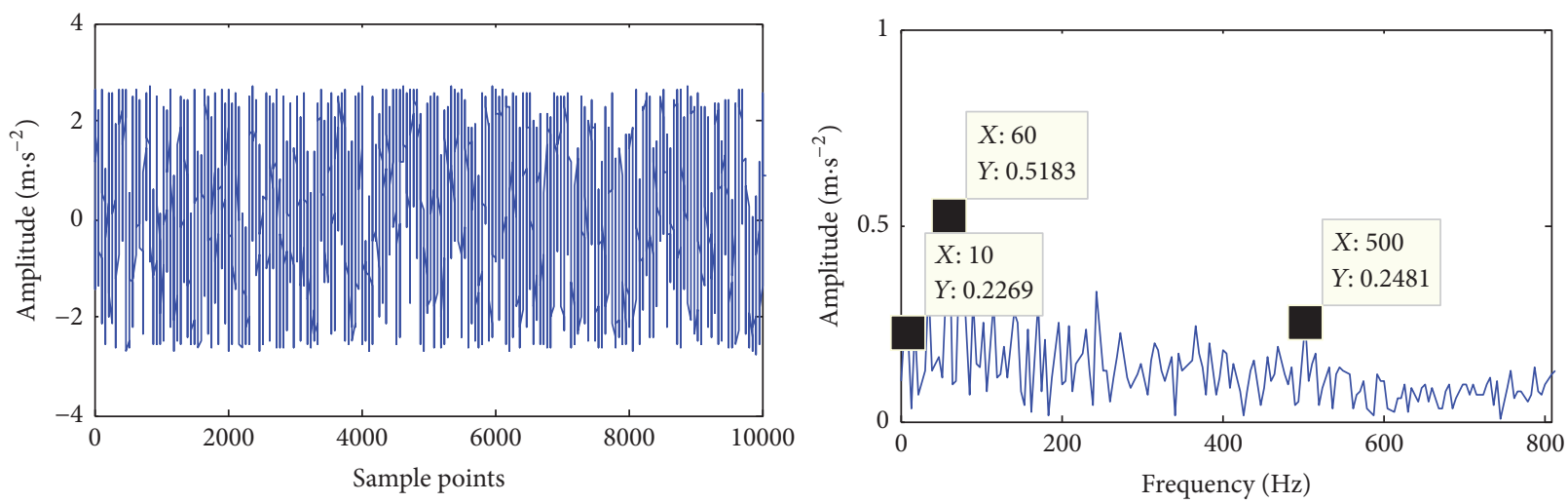

(b)
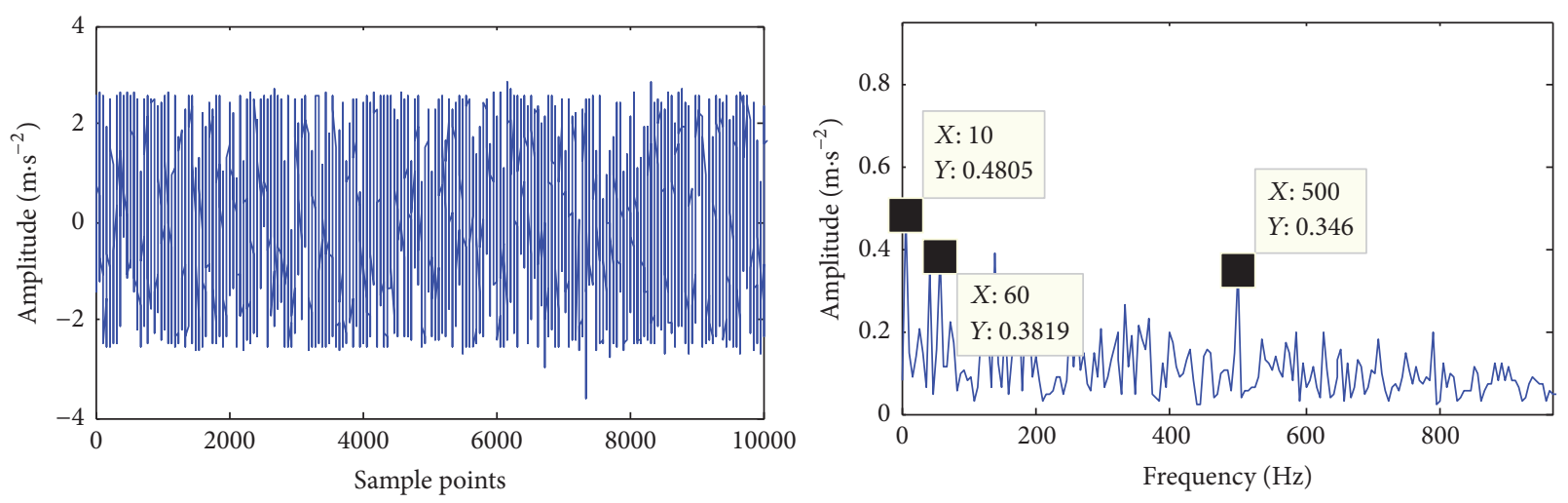

(c)

FIGURE 10: The time domain and spectrum of output signal through the rescaling multistable SR.

optimal decomposition parameters of VMD based on the minimization criterion of MESEV. In the process, the optimal decomposition parameters $\alpha$ and $k$ of VMD are set as 2000 and 3, respectively. According to the mode number $k$, the experimental data is handled using the RFMSR system with three different double sampling frequencies, namely, 1.4, 1.6, and $2 \mathrm{~Hz}$.

It can be seen from Figures 14(a)-14(c) that certain high-frequency components are weakened and the amplitude of the corresponding frequency bands is greatly improved. The amplitude of the characteristic frequency of $148.1 \mathrm{~Hz}$ increases to 0.3687 , while twice this increases to 0.1377 , and triple increases to 0.1186 . Following AMD, only the signal of each resonance band is reserved and the synthesized signal in the time and frequency domains is shown in Figure 15(a). The results of the VMD can be seen in Figures 15(b)-15(d), where it can be clearly observed that imfl, imf2, and imf 3 consist of the respective fault feature frequencies, proving the accuracy and effect of the proposed method.

3.3.2. Analysis of the Gears. In order to verify the effect of the proposed method on single-frequency diagnosis, the fault simulation in a multiple gear transmission system test bench experimental device is shown in Figure 16. Furthermore, the 


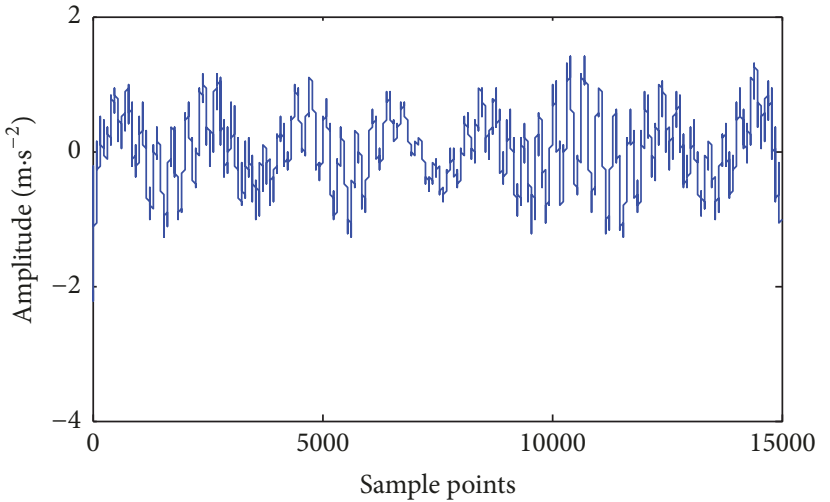

(a)

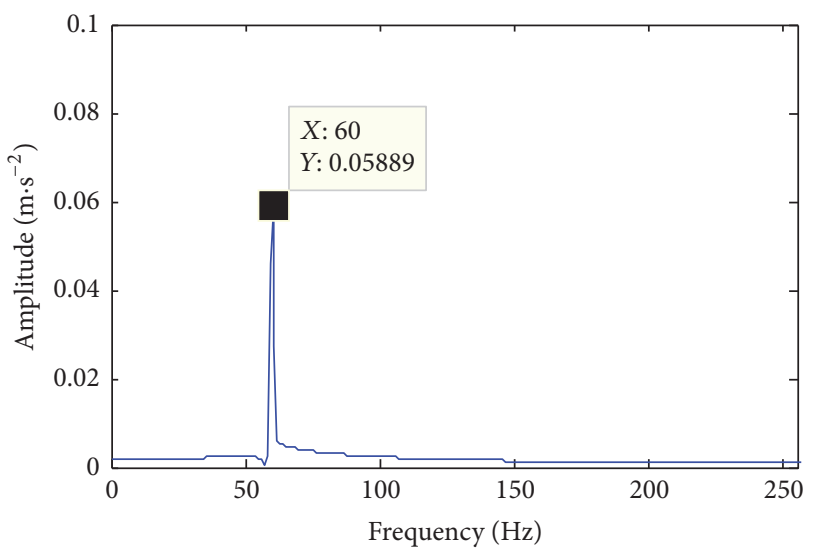

(c)

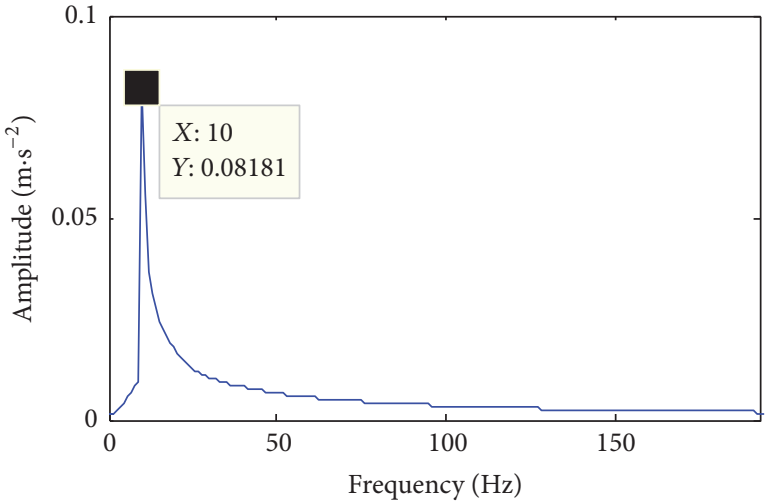

(b)

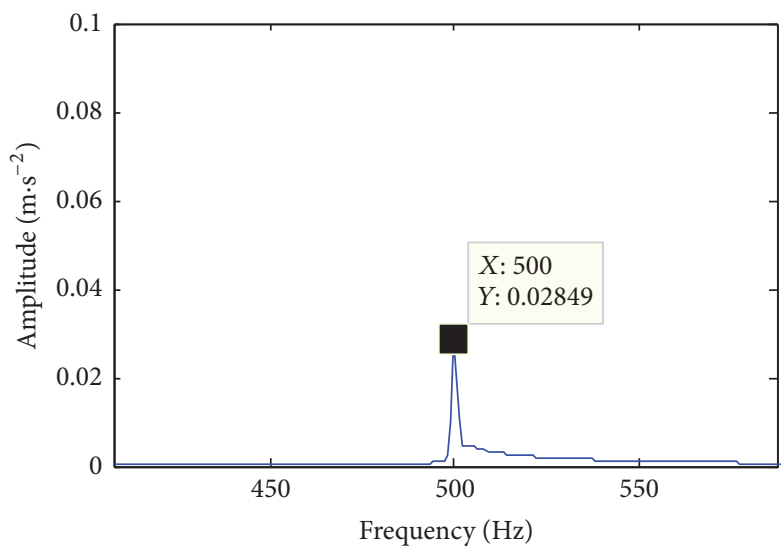

(d)

FIgURE 11: (a) The synthesized signal by AMD method and (b-d) the three IMFs of simulated signal with VMD decomposition.

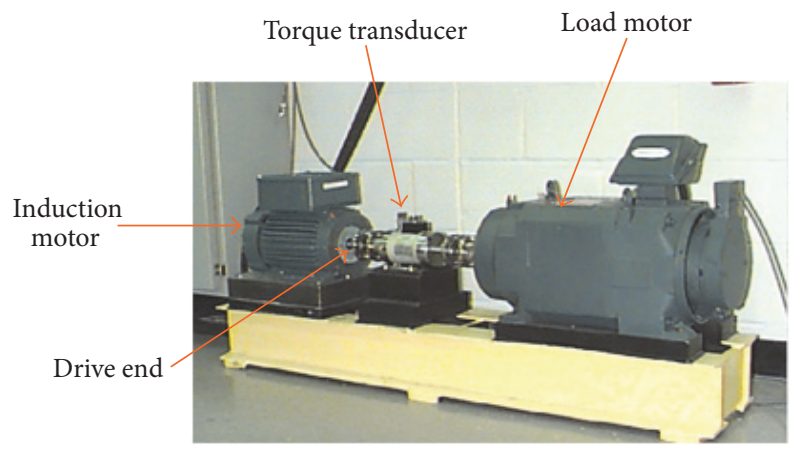

FIGURE 12: Rolling bearing fault simulation platform.

schematic diagram of the fixed axis gearbox can be seen in Figure 17. The geometric details of these types of experimental parameters are provided in Table 3 . We analysed sets of data from gear fault information using the method illustrated in Figure 8.

The number of the big gear's teeth on a low-speed shaft is 90 , while the number of the small gear's teeth on a mediumspeed shaft is 36 . And the number of big gear teeth on the
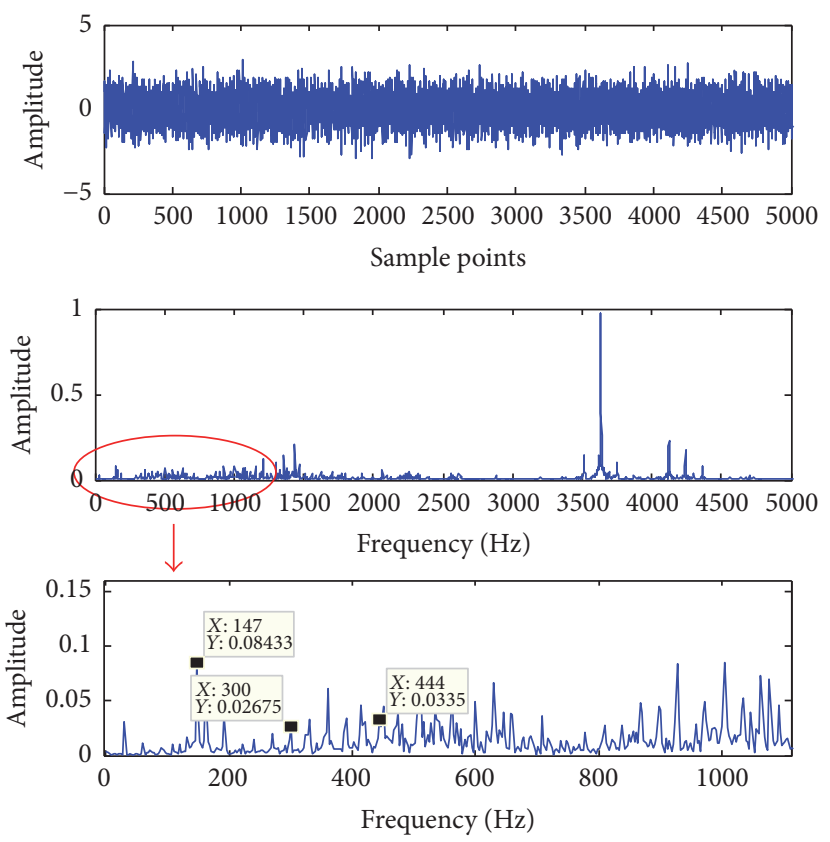

FIGURE 13: The time domain and spectrum of experimental signal. 

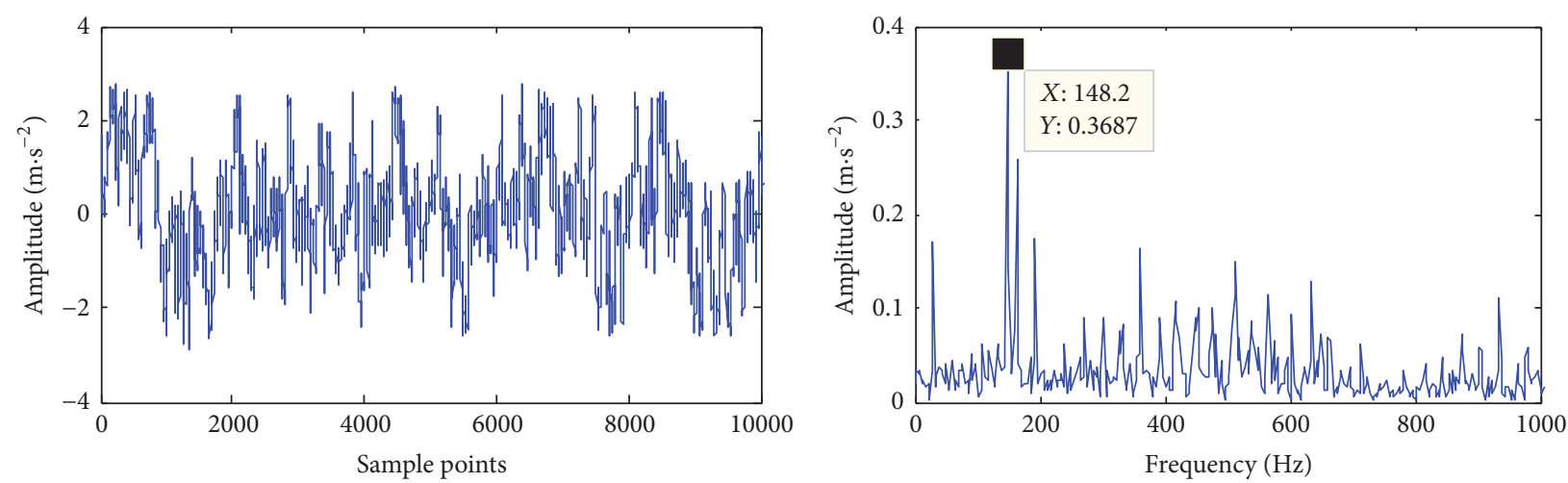

(a)
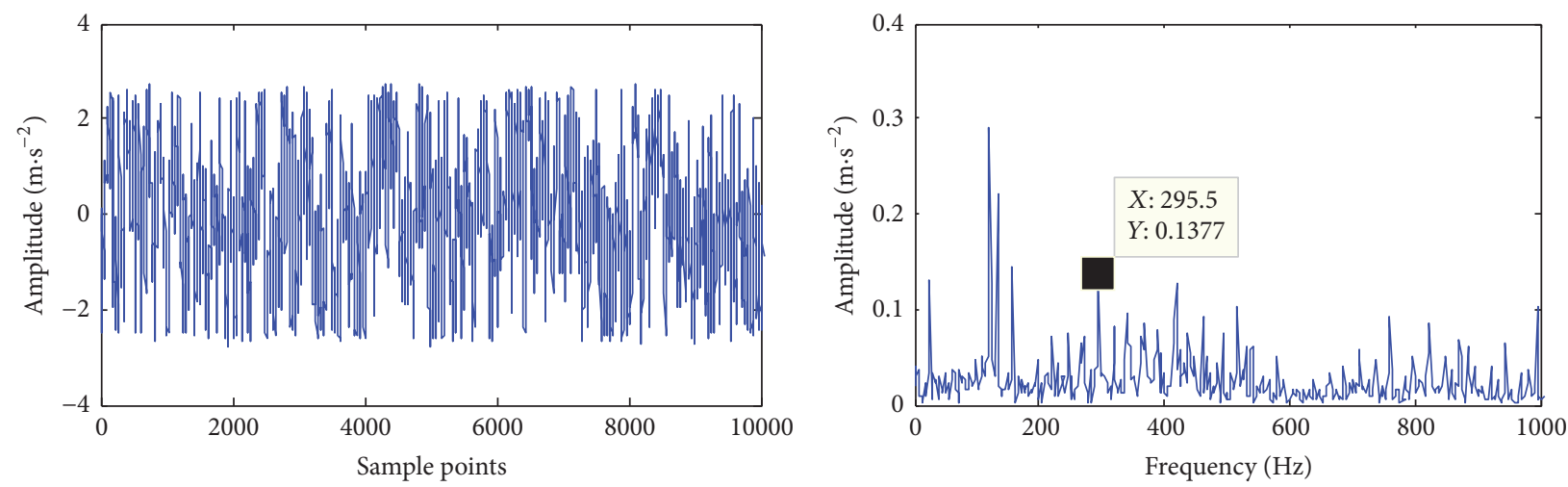

(b)
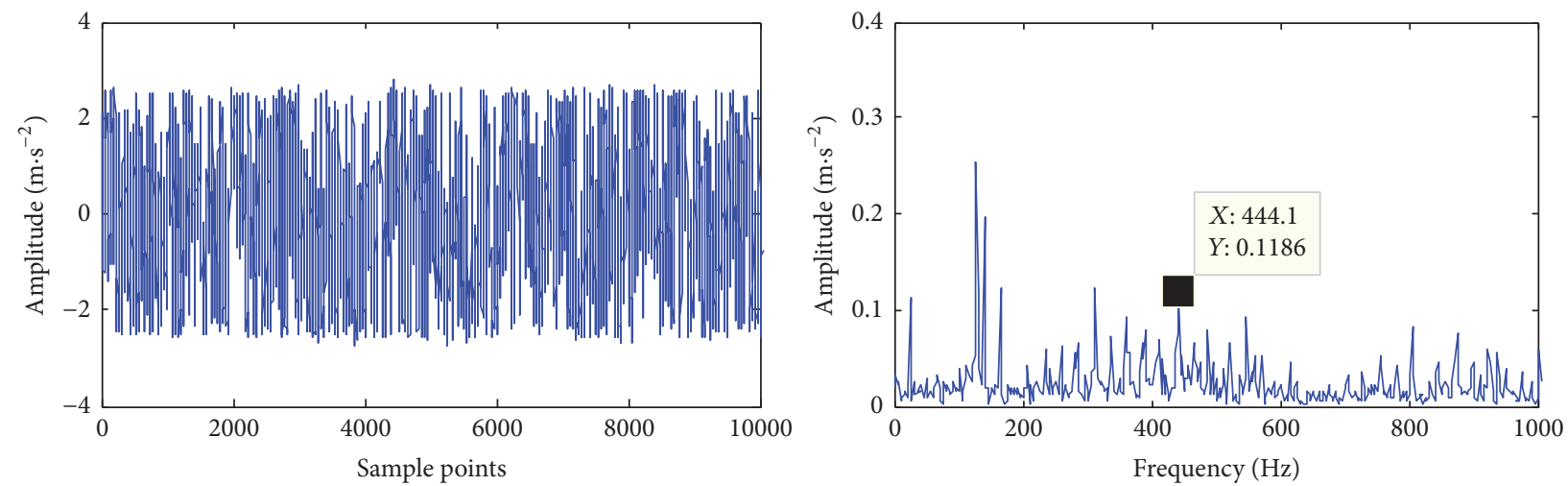

(c)

FIGURE 14: The time domain and spectrum of output signal through the rescaling multistable SR with different double sample frequency: (a) $1.4 \mathrm{~Hz}$, (b) $1.6 \mathrm{~Hz}$, and (c) $2 \mathrm{~Hz}$.

TABLE 3: Experimental parameter table.

\begin{tabular}{lc}
\hline Experimental parameters & Parameter values \\
\hline Model of experimental gear & ER-16K \\
The number of big gear's teeth on medium & 100 \\
speed shaft & $1500 \mathrm{r} / \mathrm{min}$ \\
Rotational speed & 39.408867 \\
Gear box ratio & $12 \mathrm{k} \mathrm{Hz}$ \\
Sampling frequency & $2.9 \mathrm{~Hz}$ \\
Characteristic frequency of low speed shaft &
\end{tabular}

medium-speed shaft is 100 . The meshing frequency of the gear can be calculated as follows:

$$
\begin{aligned}
f_{2} & =n_{1} \times f_{z}=n_{1} \times \frac{f_{r}}{R}=4.571 \times \frac{25}{39.408867} \\
& =2.898 \approx 2.900 \mathrm{~Hz}, \\
f_{m} & =z_{1} \times f_{1}=z_{1} \times f_{2} \times \frac{M_{1}}{M_{2}}=100 \times 2.900 \times \frac{90}{36} \\
& =725 \mathrm{~Hz},
\end{aligned}
$$




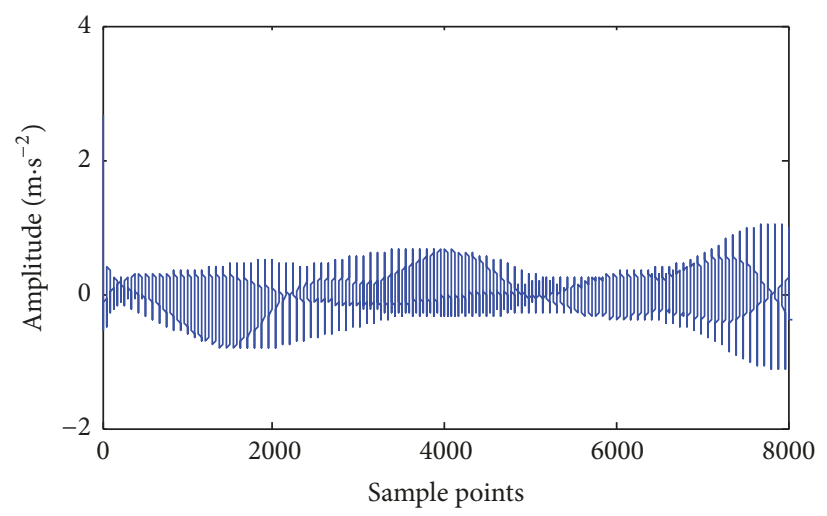

(a)

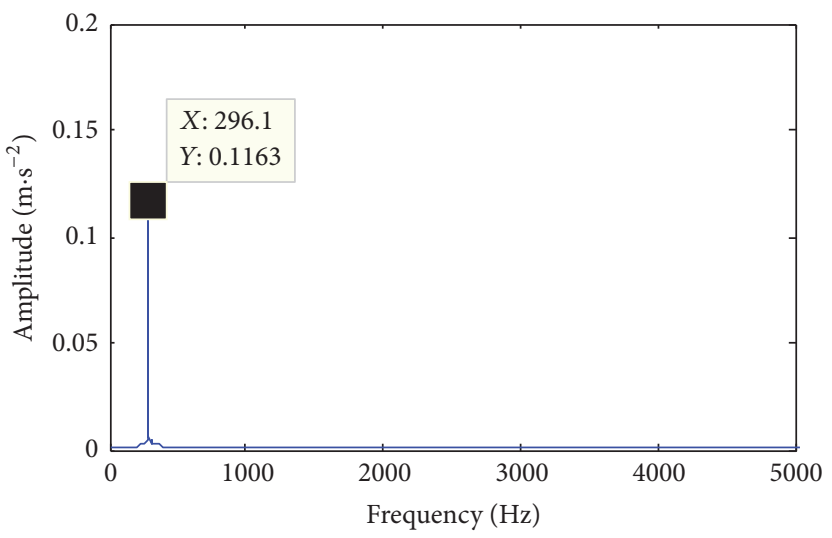

(c)

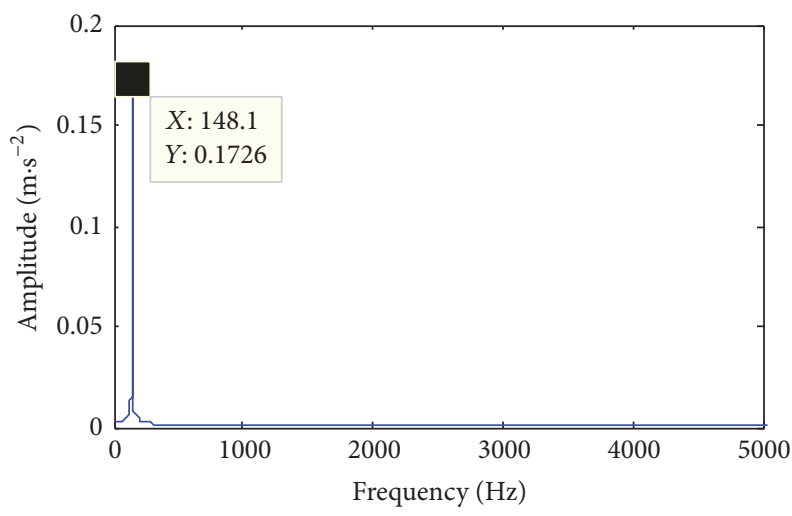

(b)

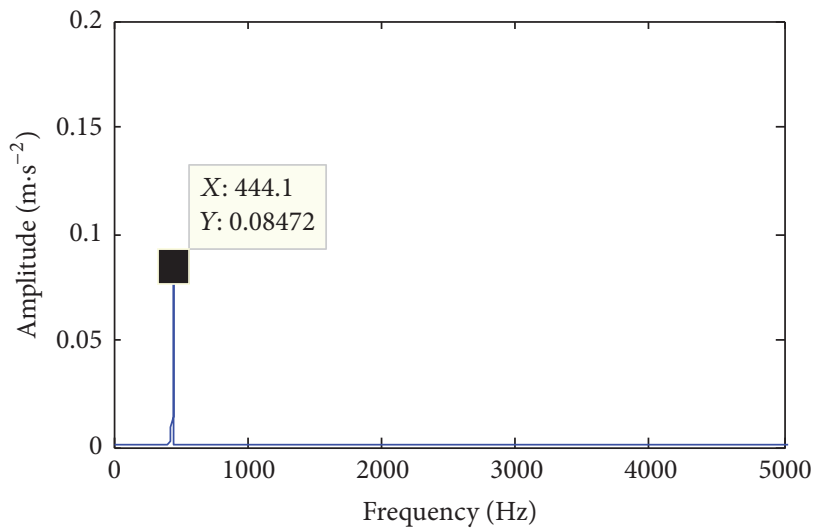

(d)

FIgURE 15: (a) The synthesized signal by AMD method and (b-d) the three IMFs of experimental signal with VMD decomposition.

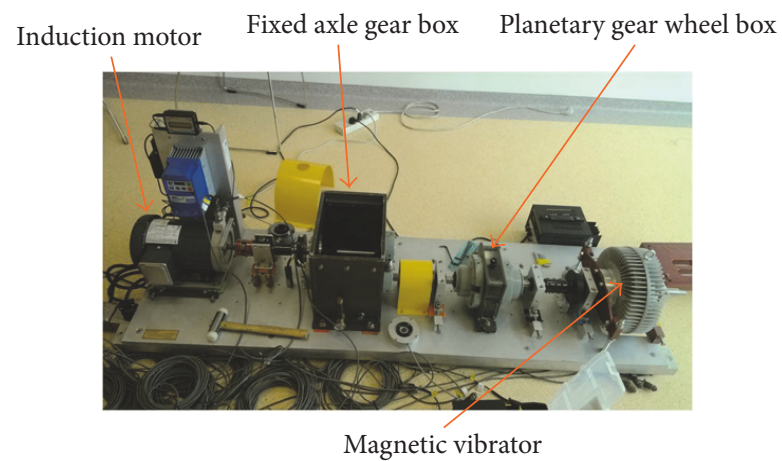

FIGURE 16: Machinery fault simulator- (MFS-) magnum experimental platform.

where $n_{1}$ is the characteristic coefficient of the low-speed shaft, $f_{z}$ is the feature frequency of the spindle, $f_{r}$ is the rotating frequency, $R$ is the gearbox ratio, $z_{1}$ represents the number of big gear teeth on the medium-speed shaft, and $M_{1}$ and $M_{2}$ represent the number of the big gear's teeth on the low-speed shaft and that of the small gear's teeth on the medium-speed shaft, respectively.

The vibration signal is measured by an accelerometer under the condition of large gear wear on the mediumspeed shaft (the area marked by the dashed-dotted red line

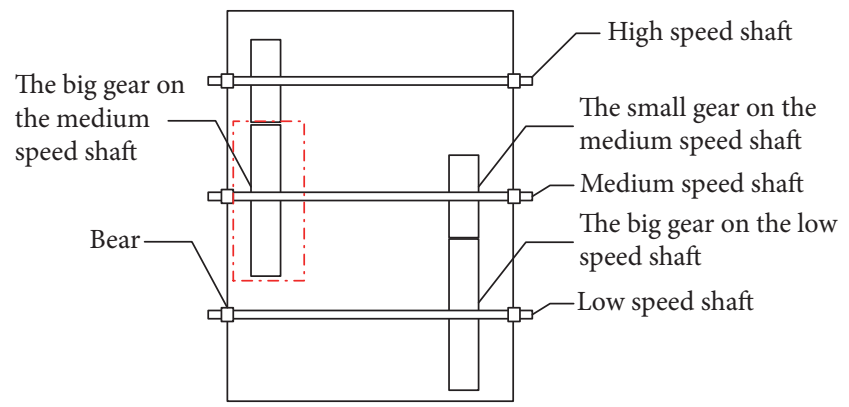

FIGURE 17: The schematic diagram of fixed axis gearbox.

in Figure 17). The gear fault signal and its spectrum diagram are illustrated in Figure 18, where it can be seen that there are obvious periodic shocks in the time domain waveform; however, the fault characteristic frequency can hardly be seen in the frequency spectrum map, because of heavy background noise interference. Therefore, we must process the gear fault signal using other effective means. The time domain waveform and frequency spectrum map of the output signals, after passing through the multistable SR system, are shown in Figure 19. Following the steps in Figure 8, the minimum entropy value appears in the twenty-seventh generation, and the best combinations of parameters $\alpha$ and $k$ are set as 

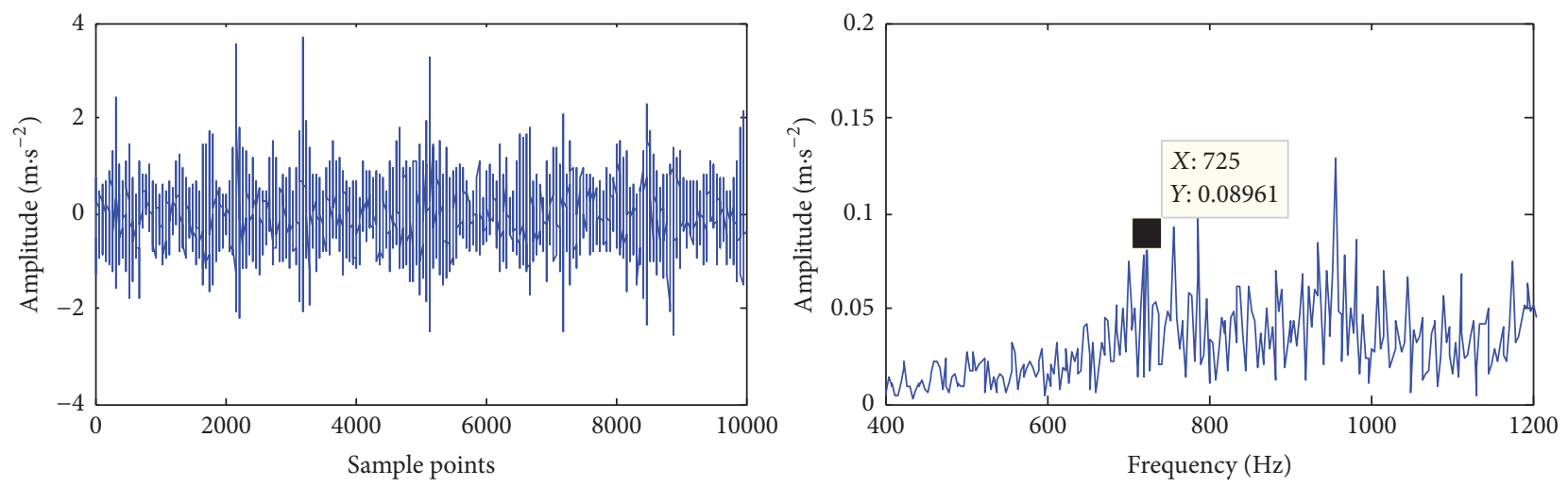

FIGURE 18: The experimental time domain signal and frequency domain signal.
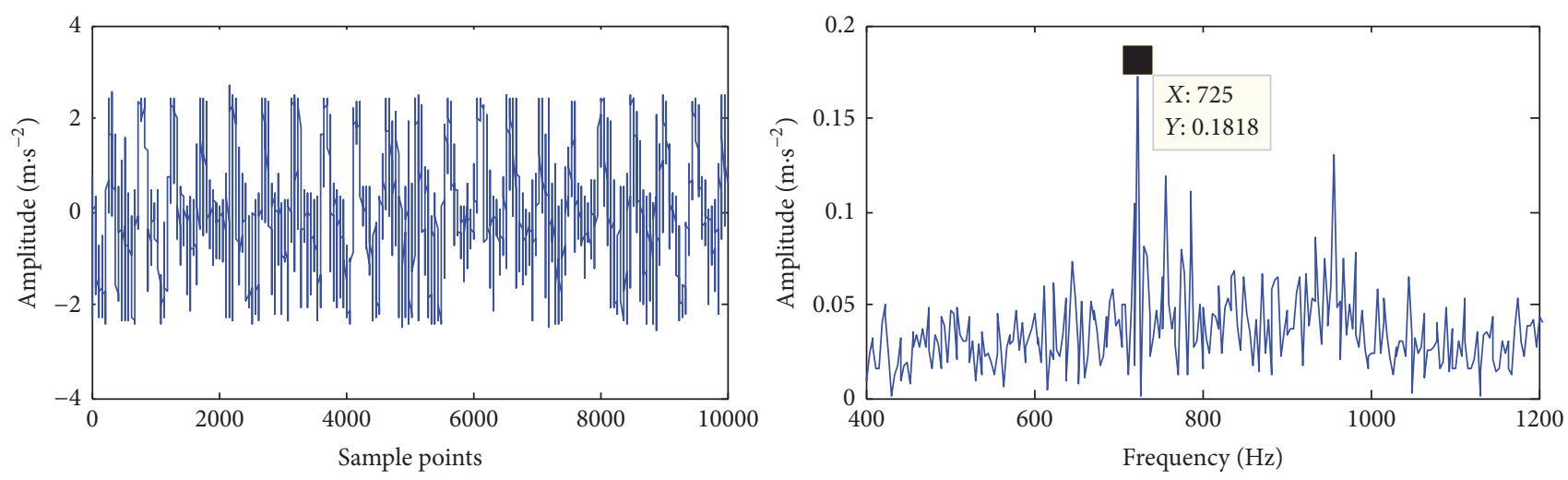

FIGURE 19: The output signal of experimental data through the rescaling multistable system.

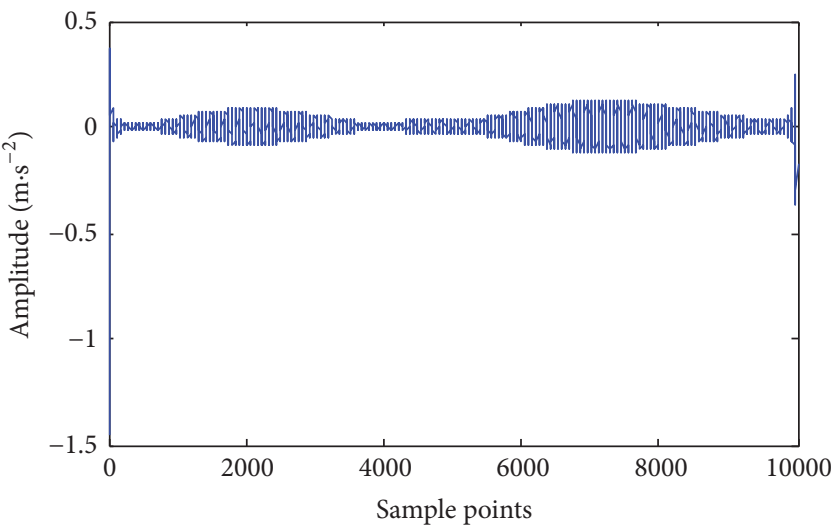

(a)

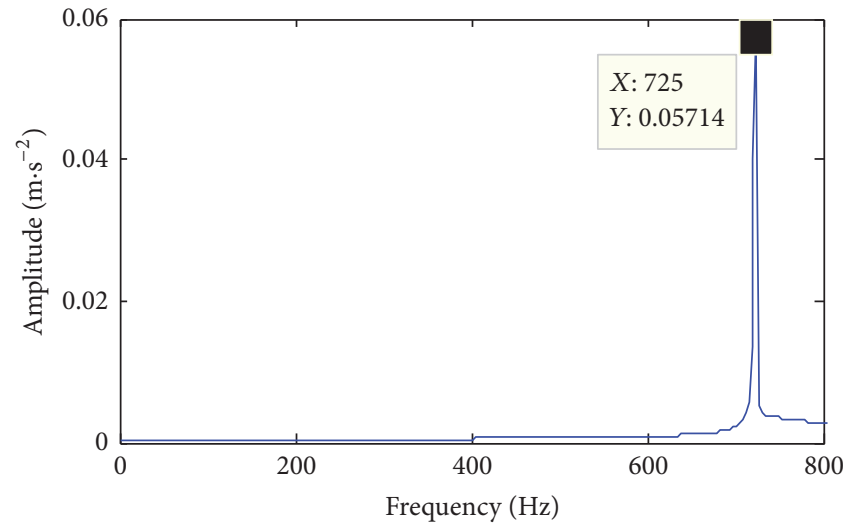

(b)

FIgURE 20: (a) The time domain of output signal by AMD. (b) The experimental signal results of VMD decomposition.

1900 and 1 . The signal in the time and frequency domains through AMD and VMD can be observed in Figure 20. It is clear that the fault feature characteristics have been extracted using the AMD and VMD methods. The experimental results demonstrate that the proposed method also proves to be accurate and practical for weak single-frequency signals.

\section{Conclusion}

In order to address the detection problems in multifrequency signals in noisy backgrounds, a novel method based on
VMD after denoising by RFMSR with AMD is proposed. The following conclusions have been reached:

(1) A rescaling frequency-shifted multistable SR with AMD can enhance the weak multifrequency signal at different scales and effectively extract useful signals in different frequency bands.

(2) Combining the RFMSR and AMD with VMD, signal noise reduction processing can be realized. Meanwhile, the RFMSR with AMD can be considered as an improved pretreatment method, prior to VMD decomposition, which solves the overdecomposition problem due to the selection 
of parameter $k$. In addition, it is beneficial for VMD to decompose the frequency components contained in the signal accurately, so as to improve the decomposition accuracy.

(3) The analysis of the simulation results and experimental examples demonstrates that this method can not only effectively detect the weak multifrequency signal under a strong noise background (including single-frequency signals), but also accurately decompose the characteristics of the signal, which further reduces the false components of VMD, improving the decomposition quality.

These conclusions provide a reliable basis for realizing weak multifrequency signal detection, and improve the shortcomings of the VMD method, particularly in a heavy noise background.

\section{Conflicts of Interest}

The authors declare that there are no conflicts of interest regarding the publication of this paper.

\section{Acknowledgments}

This work was supported by the National Natural Science Foundation of China (no. 51475407), Hebei Provincial Natural Science Foundation of China (no. E2015203190), and Key Project of Natural Science Research in Colleges and Universities of Hebei Province (Grant no. ZD2015050).

\section{References}

[1] R. B. Randall and J. Antoni, "Rolling element bearing diagnostics-a tutorial," Mechanical Systems and Signal Processing, vol. 25, no. 2, pp. 485-520, 2011.

[2] J. Liu, W. Wang, F. Golnaraghi, and K. Liu, "Wavelet spectrum analysis for bearing fault diagnostics," Measurement Science and Technology, vol. 19, no. 1, Article ID 015105, 2007.

[3] A. Martini, M. Troncossi, and A. Rivola, "Vibroacoustic measurements for detecting water leaks in buried small-diameter plastic pipes," Journal of Pipeline Systems Engineering and Practice, vol. 8, no. 4, Article ID 04017022, 2017.

[4] A. Martini, M. Troncossi, and A. Rivola, "Automatic Leak Detection in Buried Plastic Pipes of Water Supply Networks by Means of Vibration Measurements," Shock and Vibration, vol. 2015, Article ID 165304, 2015.

[5] S. Yazdekhasti, K. R. Piratla, S. Atamturktur, and A. Khan, "Experimental evaluation of a vibration-based leak detection technique for water pipelines," Structure and Infrastructure Engineering, pp. 1-10, 2017.

[6] Z. K. Peng and F. L. Chu, "Application of the wavelet transform inmachine condition monitoring and fault diagnostics: A review with bibliography," Mechanical Systems \& Signal Processing, vol. 18, pp. 199-221, 2004.

[7] J. K. Hammond and P. R. White, "The analysis of non-stationary signals using time-frequency methods," Journal of Sound and Vibration, vol. 190, no. 3, pp. 419-447, 1996.

[8] R. Yan and R. X. Gao, "Hilbert-huang transform-based vibration signal analysis for machine health monitoring," IEEE Transactions on Instrumentation and Measurement, vol. 55, no. 6, pp. 2320-2329, 2006.
[9] G. Gelle, M. Colas, and C. Servière, "Blind source separation: A new pre-processing tool for rotating machines monitoring?" IEEE Transactions on Instrumentation and Measurement, vol. 52, no. 3, pp. 790-795, 2003.

[10] A. Malhi and R. X. Gao, "PCA-based feature selection scheme for machine defect classification," IEEE Transactions on Instrumentation and Measurement, vol. 53, no. 6, pp. 1517-1525, 2004.

[11] P. Goldman and A. Muszynska, Application of Full Spectrum to Rotating Machinery Diagnostics, 1st Quarter, Bently Nevada Corporation, Minden, NV, USA, 1999.

[12] P. Shi, X. Ding, and D. Han, "Study on multi-frequency weak signal detection method based on stochastic resonance tuning by multi-scale noise," Measurement, vol. 47, no. 1, pp. 540-546, 2014.

[13] P. M. Shi, S. J. An, P. Li, and D. Y. Han, "Signal feature extraction based on cascaded multi-stable stochastic resonance denoising and EMD method," Measurement, vol. 90, pp. 318-328, 2016.

[14] W. Sun, J. Chen, and J. Li, "Decision tree and PCA-based fault diagnosis of rotating machinery," Mechanical Systems and Signal Processing, vol. 21, no. 3, pp. 1300-1317, 2007.

[15] F. Wu, T. Wang, and J. Lee, "An online adaptive condition-based maintenance method for mechanical systems," Mechanical Systems and Signal Processing, vol. 24, no. 8, pp. 2985-2995, 2010.

[16] T. Galka and M. Tabaszewski, "An application of statistical symptoms in machine condition diagnostics," Mechanical Systems Signal Processing 25, pp. 253-265, 2011.

[17] W. Guo and P. W. Tse, "Enhancing the ability of ensemble empirical mode decomposition in machine fault diagnosis," in Proceedings of the Prognostics and System Health Management Conference (PHM '10), Macau, China, January 2010.

[18] Y. G. Lei and M. J. Zuo, "Fault diagnosis of rotating machinery using an improved HHT based on EEMD and sensitive IMFs," Measurement Science and Technology, vol. 20, no. 12, Article ID 125701, 2009.

[19] K. Dragomiretskiy and D. Zosso, "Variational mode decomposition," IEEE Transactions on Signal Processing, vol. 62, no. 3, pp. 531-544, 2014.

[20] S. Mohanty, K. K. Gupta, and K. S. Raju, "Comparative study between VMD and EMD in bearing fault diagnosis," in Proceedings of the 9th IEEE International Conference on Industrial and Information Systems, ICIIS 2014, India, December 2014.

[21] Y. Wang, F. Liu, Z. Jiang, S. He, and Q. Mo, "Complex variational mode decomposition for signal processing applications," Mechanical Systems and Signal Processing, vol. 86, pp. 75-85, 2017.

[22] S. Zhang, Y. Wang, S. He, and Z. Jiang, "Bearing fault diagnosis based on variational mode decomposition and total variation denoising," Measurement Science \& Technology, vol. 27, Article ID 075101, 2016.

[23] X. Yan, M. Jia, and L. Xiang, "Compound fault diagnosis of rotating machinery based on OVMD and a 1.5-dimension envelope spectrum," Measurement Science and Technology, vol. 27, no. 7, Article ID 075002, 2016.

[24] R. Benzi, A. Sutera, and A. Vulpiani, "The mechanism of stochastic resonance," Journal of Physics A: Mathematical and General, vol. 14, no. 11, pp. L453-L457, 1981.

[25] B. Kosko and S. Mitaim, "Robust stochastic resonance: signal detection and adaptation in impulsive noise," Physical Review E, vol. 64, Article ID 051110, 2001.

[26] S. Zozor and P.-O. Amblard, "Stochastic resonance in discrete time nonlinear AR (1) models," IEEE Transactions on Signal Processing, vol. 47, no. 1, pp. 108-121, 1999. 
[27] S. Zozor and P.-O. Amblard, "Erratum: Stochastic resonance in discrete time nonlinear AR(1) models (IEEE Trans. Signal Processing (1999) 47 (108-122))," IEEE Transactions on Signal Processing, vol. 49, no. 5, pp. 1107-1109, 2001.

[28] S. Zozor and P.-O. Amblard, "On the use of stochastic resonance in sine detection," Signal Processing, vol. 82, no. 3, pp. 353-367, 2002.

[29] F. Duan, F. Chapeau-Blondeau, and D. Abbott, "Fisherinformation condition for enhanced signal detection via stochastic resonance," Physical Review E: Statistical, Nonlinear, and Soft Matter Physics, vol. 84, no. 5, Article ID 051107, 2011.

[30] V. N. Hari, G. V. Anand, A. B. Premkumar, and A. S. Madhukumar, "Design and performance analysis of a signal detector based on suprathreshold stochastic resonance," Signal Processing, vol. 92, no. 7, pp. 1745-1757, 2012.

[31] F. Duan and D. Abbott, "Signal detection for frequency-shift keying via short-time stochastic resonance," Physics Letters A, vol. 344, no. 6, pp. 401-410, 2005.

[32] F. Duan and D. Abbott, "Binary modulated signal detection in a bistable receiver with stochastic resonance," Physica A: Statistical Mechanics and its Applications, vol. 376, no. 1-2, pp. 173-190, 2007.

[33] F. Chapeau-Blondeau, F. Duan, and D. Abbott, "Signal-tonoise ratio of a dynamical saturating system: Switching from stochastic resonator to signal processor," Physica A: Statistical Mechanics and its Applications, vol. 387, no. 11, pp. 2394-2402, 2008.

[34] F. Duan, F. Chapeau-Blondeau, and D. Abbott, "Exploring weak-periodic-signal stochastic resonance in locally optimal processors with a Fisher information metric," Signal Processing, vol. 92, no. 12, pp. 3049-3055, 2012.

[35] J. Y. Tan, X. F. Chen, and J. Y. Wang, "Study of frequency-shifted and re-scaling stochastic resonance and its application to fault diagnosis," Mechanical Systems and Signal Processing, vol. 23, no. 3, pp. 811-822, 2009.

[36] Y. G. Leng, Y. S. Leng, T. Y. Wang, and Y. Guo, "Numerical analysis and engineering application of large parameter stochastic resonance," Journal of Sound and Vibration, vol. 292, no. 3-5, pp. 788-801, 2006.

[37] D. Han, P. li, S. An, and P. Shi, "Multi-frequency weak signal detection based on wavelet transform and parameter compensation band-pass multi-stable stochastic resonance," Mechanical Systems and Signal Processing, vol. 70-71, pp. 995-1010, 2016.

[38] Q. Mao, M. Lin, and Y. Zheng, "Study of weak multi-frequency signal detection based on stochastic resonance," Journal of Basic Science and Engineering, vol. 16, pp. 86-91, 2008.

[39] G. Chen and Z. Wang, "A signal decomposition theorem with Hilbert transform and its application to narrowband time series with closely spaced frequency components," Mechanical Systems and Signal Processing, vol. 28, pp. 258-279, 2012.

[40] P. Shi, C. Su, and D. Han, "Fault diagnosis of rotating machinery based on adaptive stochastic resonance and AMD-EEMD," Shock and Vibration, vol. 2016, Article ID 9278581, 11 pages, 2016.

[41] M. Feldman, "A signal decomposition or lowpass filtering with Hilbert transform?" Mechanical Systems and Signal Processing, vol. 25, no. 8, pp. 3205-3208, 2011.

[42] M. R. Hestenes, "Multiplier and gradient methods," Journal of Optimization Theory and Applications, vol. 4, pp. 303-320, 1969.

[43] D. E. Goldberg, Genetic Algorithm in Search, Optimization, and Machine Learning, 1989.
[44] K. Deb, A. Pratap, S. Agarwal, and T. Meyarivan, "A fast and elitist multiobjective genetic algorithm: NSGA-II," IEEE Transactions on Evolutionary Computation, vol. 6, no. 2, pp. 182197, 2002. 


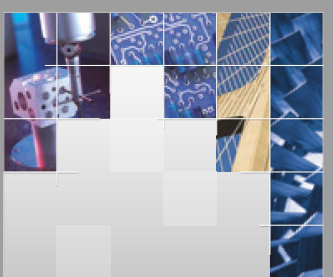

\section{Enfincering}
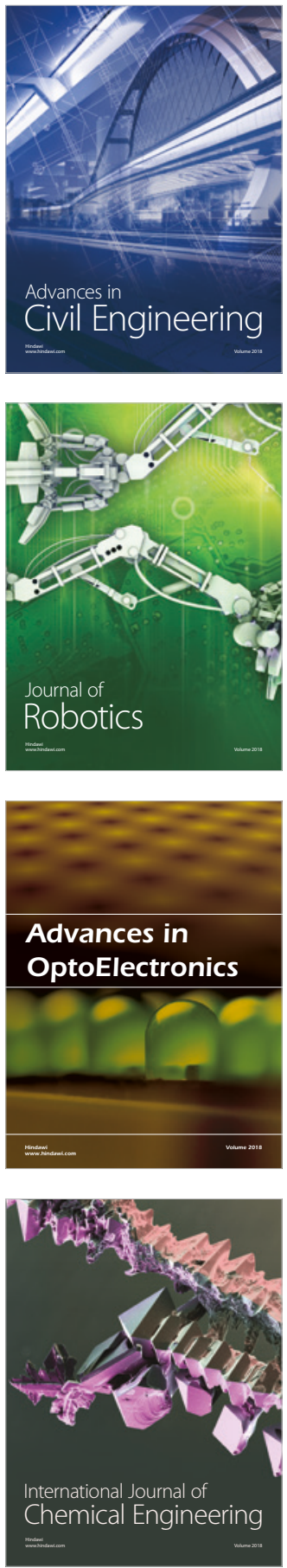

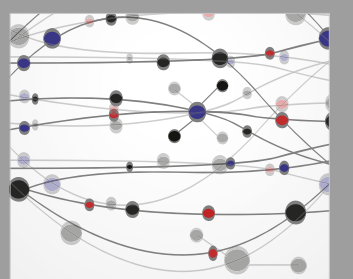

\section{Rotating \\ Machinery}

The Scientific World Journal

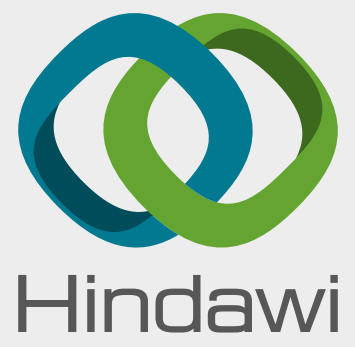

Submit your manuscripts at

www.hindawi.com
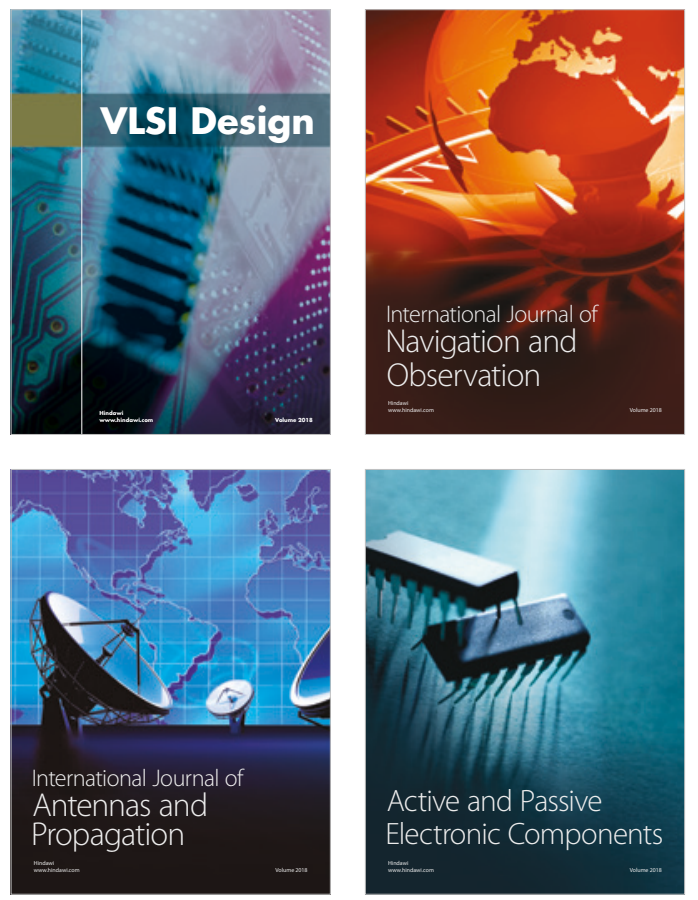
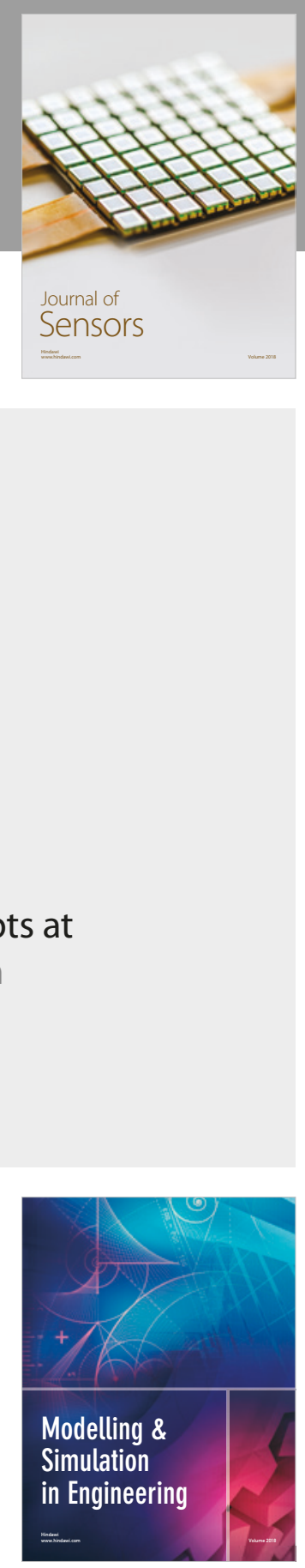

\section{Advances \\ Multimedia}
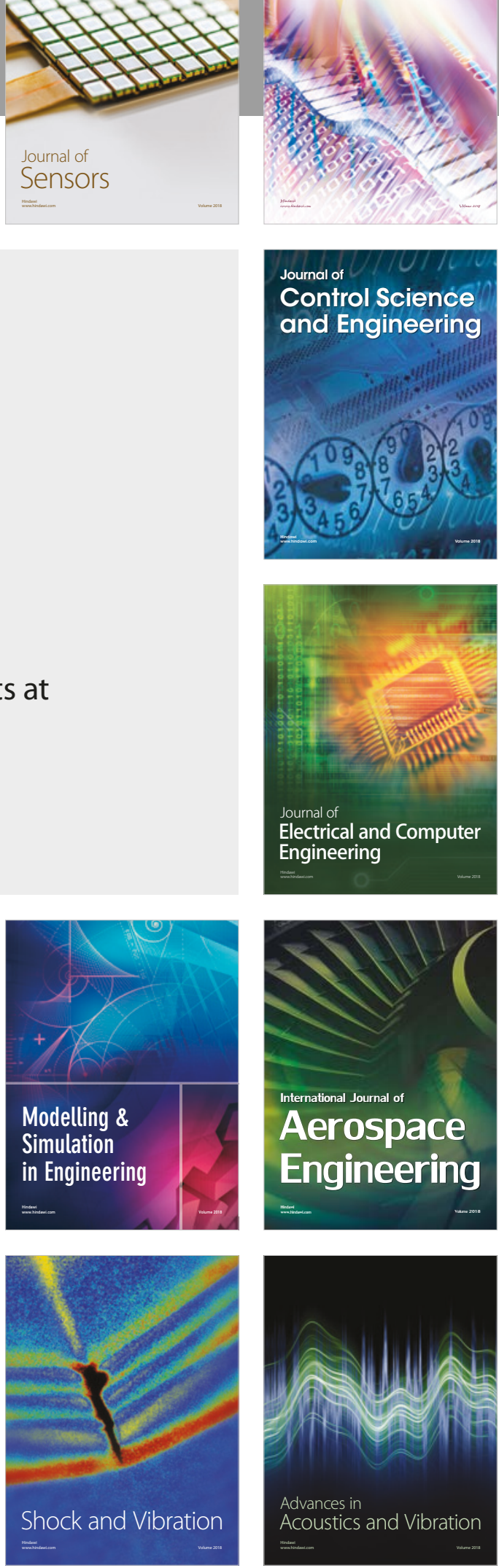